\title{
Fast Multiqubit Gates through Simultaneous Two-Qubit Gates
}

\author{
Xiu Gu๑, ${ }^{1,2, *, \sharp ~ J o r g e ~ F e r n a ́ n d e z-P e n d a ́ s ~} \odot,{ }^{1}$ Pontus Vikstålø, ${ }^{1}$ Tahereh Abad, ${ }^{1}$ Christopher Warren $\odot,{ }^{1}$ \\ Andreas Bengtsson $\odot,{ }^{1}$ Giovanna Tancredi, ${ }^{1}$ Vitaly Shumeiko $\odot,{ }^{1}$ Jonas Bylander $\odot,{ }^{1}$ \\ Göran Johansson, ${ }^{1}$ and Anton Frisk Kockum ${ }^{1, \dagger}$ \\ ${ }^{1}$ Department of Microtechnology and Nanoscience, Chalmers University of Technology, 412 96, \\ Gothenburg, Sweden \\ ${ }^{2}$ Tencent Quantum Laboratory, Tencent, Shenzhen, Guangdong 518057, China
}

(Received 4 July 2021; accepted 8 November 2021; published 8 December 2021)

\begin{abstract}
Near-term quantum computers are limited by the decoherence of qubits to only being able to run lowdepth quantum circuits with acceptable fidelity. This severely restricts what quantum algorithms can be compiled and implemented on such devices. One way to overcome these limitations is to expand the available gate set from single- and two-qubit gates to multiqubit gates, which entangle three or more qubits in a single step. Here, we show that such multiqubit gates can be realized by the simultaneous application of multiple two-qubit gates to a group of qubits where at least one qubit is involved in two or more of the two-qubit gates. Multiqubit gates implemented in this way are as fast as, or sometimes even faster than, the constituent two-qubit gates. Furthermore, these multiqubit gates do not require any modification of the quantum processor, but are ready to be used in current quantum-computing platforms. We demonstrate this idea for two specific cases: simultaneous controlled- $Z$ gates and simultaneous isWAP gates. We show how the resulting multiqubit gates relate to other well-known multiqubit gates and demonstrate through numerical simulations that they would work well in available quantum hardware, reaching gate fidelities well above $99 \%$. We also present schemes for using these simultaneous two-qubit gates to swiftly create large entangled states like Dicke and Greenberger-Horne-Zeilinger states.
\end{abstract}

DOI: 10.1103/PRXQuantum.2.040348

\section{INTRODUCTION}

Quantum computers [1,2] hold a promise of eventually being able to tackle complex problems in chemistry $[3,4]$, materials science [5], finance [6,7], simulation of quantum systems [8], and many other fields [9-12]. However, current [13-17] and near-term quantum computers are noisy intermediate-scale quantum (NISQ) [11] devices, where decoherence leads to loss of entanglement and coherence among the qubits in the quantum computer after a relatively short time. Thus, such devices can only run quantum circuits with a low depth, i.e., consisting of a low number of sequential quantum gates.

All quantum algorithms can be decomposed into a sequence of universal single- and two-qubit gates $[2,18]$.

\footnotetext{
*guxiu1@gmail.com

†anton.frisk.kockum@chalmers.se

\$Present address: Tencent Quantum Laboratory, Tencent, Shenzhen, Guangdong 518057, China.

Published by the American Physical Society under the terms of the Creative Commons Attribution 4.0 International license. Further distribution of this work must maintain attribution to the author(s) and the published article's title, journal citation, and DOI.
}

Current quantum computers are usually able to implement a universal gate set with arbitrary single-qubit rotations and one or two entangling two-qubit gates. However, many quantum algorithms, e.g., for optimization problems or quantum simulations, require the creation of large-scale entanglement or many-body interactions. Such interactions between three or more qubits result in a large overhead in terms of circuit depth if they are to be decomposed into and compiled from two-qubit gates [2]. For example, decomposing the three-qubit Fredkin gate requires at least five two-qubit gates [19].

Motivated by these limitations of NISQ devices, there has recently been several proposals [20-37] for and some implementations [38-43] of multiqubit gates, as well as proposals for realizing many-body interactions [44-50], without having to decompose them into two-qubit gates. However, in general, these proposals and implementations require one or more of the following: additional resources (e.g., extra qubits, modes, energy levels, or initial entanglement); specific complicated connectivity between qubits; setups or components that go beyond what is needed for implementing single- and two-qubit gates; complicated pulse shapes (which, in superconducting circuits, can be distorted due to the response function of drive lines and limited time resolution of arbitrary waveform 
generators [51]); or phenomena specific to a particular quantum-computing platform.

In this article, we show how various multiqubit gates can be constructed by simply applying multiple two-qubit gates simultaneously to several qubits such that at least one of the qubits is involved in two or more of the twoqubit gates. The multiqubit gates we propose can thus be implemented in existing quantum hardware adapted to standard single- and two-qubit gates, without any additional components, complicated pulse shapes, or changes in hardware design being required. Furthermore, our multiqubit gates are as fast as, or faster than, the two-qubit gates from which they are constructed. Although our examples and discussion of experimental feasibility focus on implementations of quantum computing in superconducting circuits $[10,13,15,52-56]$, our ideas are applicable to any other quantum-computing platforms, e.g., trapped ions [14,57-62] and Rydberg atoms [63-67], that either implement two-qubit gates in similar ways or have other native two-qubit gates that can be run simultaneously to create new multiqubit gates. The results presented here thus open up avenues for speeding up quantum computation across many different algorithms and systems.

We illustrate our general idea with two specific examples, simultaneous controlled- $Z(\mathrm{CZ})$ gates and simultaneous iSWAP gates, but note that the simultaneous application of other gates should also be explored. In the first example, we consider $\mathrm{CZ}$ gates created by activating the transition between states $|11\rangle$ and $|02\rangle$ (or $|20\rangle$ ) [68], where $|0\rangle$ is the ground state and $|1\rangle$ is the first excited state of a qubit, and $|2\rangle$ is the second excited state, which typically is outside the computational subspace. Activating the interaction required for two such gates simultaneously between the nearest neighbors in a linear chain of three qubits, with the middle qubit being the one where the second excited state $|2\rangle$ is populated during the gates, results in a threequbit gate where both CZ and SWAP gates are applied to the outer qubits conditioned on the middle qubit being in $|1\rangle$. This three-qubit gate, which we denote CCZS [69], takes less time than a single $\mathrm{CZ}$ gate and would require at least three sequential two-qubit gates if it were to be decomposed. The well-known three-qubit iFredkin gate [70] can be realized by adding a two-qubit gate after the CCZS gate. Furthermore, by changing the relative strengths of the constituent $\mathrm{CZ}$ gates and their detuning, a whole family of three-qubit gates can be created. These gates can be used to create many-qubit entangled states, e.g., a GreenbergerHorne-Zeilinger (GHZ) state [71,72] in a single step or large Dicke states [73-75] in a few steps, and have applications in phase estimation [2,76], Hamiltonian simulation [77-79], quantum singular value transformation [80-82], and swap tests [83] for quantum machine learning [84].

In our second example, we consider isWAP gates created by coupling the states $|01\rangle$ and $|10\rangle$. Just like for the CCZS gate above, simultaneous activation of the interaction for two such gates in a linear chain of three qubits creates a three-qubit gate, which we denote DIV for "divider" gate. The DIV gate distributes excitations among all three qubits within the subspaces with fixed excitation number in the computational subspace. Similar to the CCZS gate, the DIV gate is faster than the two-qubit gates created by activating the same interactions and can be used to create both GHZ and large Dicke states. By changing the relative strengths of the constituent isWAP gates and the gate time, a family of different three-qubit DIV gates can be realized. Since both the DIV gates and the CCZS gates conserve the number of excitations, they may find applications in quantum-chemistry calculations with a fixed number of electrons [85] or in the mixing layer of the quantum alternating operator ansatz [86] for constrained combinatorial optimization with conserved Hamming weights [87].

This article is organized as follows. In Sec. II, we present the details for generating a family of multiqubit gates through simultaneous application of multiple $\mathrm{CZ}$ gates. We show how this family of three-qubit gates can be decomposed into a sequence of three two-qubit gates and how the three-qubit gates can be used to implement other well-known three-qubit gates through some additional operation. We then present schemes for rapidly generating large entangled states using our three-qubit gates. Finally, we show, through numerical simulations with parameters from state-of-the-art superconducting quantum-computing platforms, that our three-qubit gates are ready to be implemented with high fidelity and short gate times in currently available quantum hardware. In Sec. III, we repeat these steps for simultaneous application of isWAP gates instead of CZ gates. We conclude in Sec. IV and give an outlook for future work and applications in Sec. V. Some further analytical calculations for the simultaneous $\mathrm{CZ}$ gates with an additional coupling between the outer qubits in the linear chain are given in Appendix A and additional data from the numerical simulations of possible experimental applications are presented in Appendix B.

\section{SIMULTANEOUS CONTROLLED-Z GATES}

\section{A. Setup and gate operation}

We here consider simultaneous application of $\mathrm{CZ}$ gates that are based on making the states $|11\rangle$ and $|02\rangle$ (or $|20\rangle$ ) resonant [68]. In such gates, the states $|00\rangle,|01\rangle$, and $|10\rangle$ do not couple to other states and remain unchanged while the state $|11\rangle$ acquires a $\pi$ phase shift when its population is transferred to $|02\rangle$ (or $|20\rangle$ ) and back. In superconducting circuits, this can be achieved either by rapidly tuning the frequencies of the two qubits in and out of the desired resonance [88-94] or by parametric modulation of a coupler connecting the two qubits activating the interaction between $|11\rangle$ and $|02\rangle$ (or $|20\rangle$ ) [95-98]. For both methods, high gate fidelities have been demonstrated for short gate 
(a)

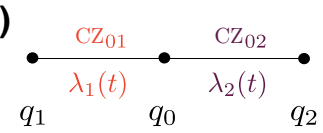

(c)

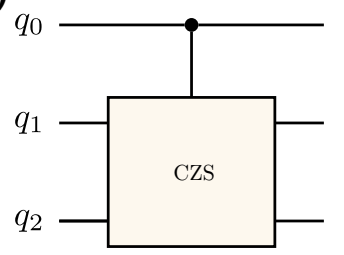

(b)

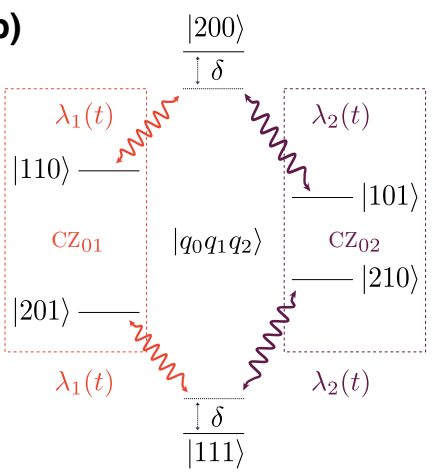

FIG. 1. Setup and operation for the three-qubit gate realized through simultaneous application of two CZ gates. (a) The setup considered is a linear chain of three qubits with nearest-neighbor coupling. Going from left to right in the chain, we denote the qubits $q_{1}, q_{0}$, and $q_{2}$. The $\mathrm{CZ}$ gates $\mathrm{CZ}_{0 j}$ between qubits 0 and $j=\{1,2\}$ are applied simultaneously by activating a coupling between the $\left|1_{0} 1_{j}\right\rangle$ and $\left|2_{0} 0_{j}\right\rangle$ states with the coupling strength $\lambda_{j}(t)$. (b) The transitions in the three-qubit system activated by the application of the $\mathrm{CZ}$ gates. With the three-qubit states denoted by $\left|q_{0} q_{1} q_{2}\right\rangle$, the transitions $|11 x\rangle \leftrightarrow|20 x\rangle$ with $x=$ $\{0,1\}$ are activated by $\mathrm{CZ}_{01}$ (red), and the transitions $|1 \times 1\rangle \leftrightarrow$ $|2 x 0\rangle$ are activated by $\mathrm{CZ}_{02}$ (purple). We assume that both $\mathrm{CZ}$ gate operations are detuned by $\delta$ from resonance. (c) We denote the three-qubit operation resulting from the simultaneous application of the two CZ gates by CCZS (controlled-CZS), since it applies both $\mathrm{CZ}$ and SWAP gates to the target qubits $q_{1}$ and $q_{2}$ conditioned on the control qubit $q_{0}$.

times. In Ref. [91], a gate fidelity of $99.9 \%$ was reached for a gate time of $60 \mathrm{~ns}$.

\section{Hamiltonians and time evolution}

We first treat the case of three qubits with simultaneous application of two CZ gates (the case of more qubits is discussed further in Sec. II D below). We consider the setup shown in Fig. 1(a), with the three qubits arranged in a linear chain such that qubit $q_{0}$ is in the middle, qubit $q_{1}$ is on the left, and qubit $q_{2}$ is on the right. The more complicated case with an additional direct coupling existing between $q_{1}$ and $q_{2}$ is discussed in Appendix A.

In the setup of Fig. 1(a), the transitions between states $\left|1_{0} 1_{j}\right\rangle$ and $\left|2_{0} 0_{j}\right\rangle$ are coupled with a strength $\lambda_{j}(t)$ to implement the standard two-qubit gates $\mathrm{CZ}_{0 j}$ between qubits 0 and $j=\{1,2\}$ by activating the coupling for a time corresponding to a complete transfer of population from $\left|1_{0} 1_{j}\right\rangle$ to $\left|2_{0} 0_{j}\right\rangle$ and back. If both these $\mathrm{CZ}$ gates are applied simultaneously, transitions $|110\rangle \leftrightarrow|200\rangle \leftrightarrow$ $|101\rangle$ and $|201\rangle \leftrightarrow|111\rangle \leftrightarrow|210\rangle$, where the states are ordered as $\left|q_{0} q_{1} q_{2}\right\rangle$, are activated. This creates a $\Lambda$-type three-level system and a $V$-type three-level system, as shown in Fig. 1(b).

With all other transitions except those shown in Fig. 1(b) far off resonance, the Hamiltonian for the three-qubit system can be written in the interaction picture as $(\hbar=1$ throughout this article)

$$
\begin{aligned}
H= & {\left[\lambda_{1}(t)(|110\rangle\langle 200|+| 111\rangle\langle 201|)\right.} \\
& \left.+\lambda_{2}(t)(|101\rangle\langle 200|+| 111\rangle\langle 210|)+\text { H.c. }\right] \\
& +\delta(|200\rangle\langle 200|-| 111\rangle\langle 111|),
\end{aligned}
$$

where H.c. denotes the Hermitian conjugate and $\delta$ is the detuning, assumed to be the same, for the transitions of both $\mathrm{CZ}$ gates. To analyze the time evolution generated by $H$, it is convenient to deal with the two effective three-level systems in Fig. 1(b) separately.

For the effective $\Lambda$-type three-level system, i.e., the subspace spanned by $|101\rangle,|200\rangle$, and $|110\rangle$, we can introduce a new basis: the bright state $|B\rangle$, the dark state $|D\rangle$, and the excited state $|E\rangle$. These states are given by

$$
\begin{aligned}
& |B\rangle=e^{i \phi} \sin \frac{\theta}{2}|101\rangle-\cos \frac{\theta}{2}|110\rangle, \\
& |D\rangle=\cos \frac{\theta}{2}|101\rangle+e^{-i \phi} \sin \frac{\theta}{2}|110\rangle, \\
& |E\rangle=|200\rangle,
\end{aligned}
$$

with

$$
\frac{\lambda_{2}(t)}{\lambda_{1}(t)}=-e^{i \phi} \tan \frac{\theta}{2}
$$

In this basis, the Hamiltonian of this subspace becomes

$$
\begin{aligned}
H_{+} & =(\Omega|B\rangle\langle E|+\text { H.c. })+\delta|E\rangle\langle E| \\
& =\Omega \sigma_{x}^{(B, E)}-\frac{\delta}{2} \sigma_{z}^{(\mathrm{B}, \mathrm{E})}+\frac{\delta}{2} I^{(B, E)},
\end{aligned}
$$

where

$$
\Omega=\sqrt{\left|\lambda_{1}(t)\right|^{2}+\left|\lambda_{2}(t)\right|^{2}}
$$

and the $\sigma_{i}^{(B, E)}$ are the Pauli matrices in the basis of $|B\rangle$ and $|E\rangle$.

We now consider the simple case where $\lambda_{1}, \lambda_{2}$, and $\delta$ are time independent. In that case, the time evolution for the three-level system only affects the two-level subspace spanned by $|B\rangle$ and $|E\rangle$. The time-evolution operator becomes

$$
\begin{aligned}
U^{(B, E)}(t)= & e^{-i \delta t / 2}\left[\cos \left(t \sqrt{\Omega^{2}+\frac{\delta^{2}}{4}}\right)\right. \\
& \left.-i \sin \left(t \sqrt{\Omega^{2}+\frac{\delta^{2}}{4}}\right) \vec{n}^{(B, E)} \cdot \vec{\sigma}^{(B, E)}\right],
\end{aligned}
$$


where

$$
\vec{n}^{(B, E)}=\frac{1}{\sqrt{\Omega^{2}+\delta^{2} / 4}}\left(\Omega, 0,-\frac{\delta}{2}\right) .
$$

For this time evolution to yield a useful gate, we need to eliminate any leakage to the state $|E\rangle=|200\rangle$, since it is outside the computational subspace. When starting in the computational subspace, the shortest evolution time that fulfils this condition is

$$
t_{\mathrm{gate}}=\frac{\pi}{\sqrt{\Omega^{2}+\delta^{2} / 4}} .
$$

After this time, the states $|B\rangle$ and $|E\rangle$ both acquire a phase factor $-e^{-i \gamma}$, where

$$
\gamma=\frac{\pi \delta}{\sqrt{4 \Omega^{2}+\delta^{2}}}
$$

while the dark state $|D\rangle$ remains unchanged. Since $|B\rangle$ and $|D\rangle$ also constitute a basis for the subspace spanned by $|101\rangle$ and $|110\rangle$, the effect of the time evolution can be written as $[99,100]$

$$
|D\rangle\left\langle D\left|-e^{-i \gamma}\right| B\right\rangle\langle B|=e^{(i / 2)(\pi-\gamma)} e^{-(i / 2)(\pi-\gamma) \vec{n} \cdot \vec{\sigma}},
$$

where

$$
\begin{aligned}
\vec{n} & =(\sin \theta \cos \phi, \sin \theta \sin \phi, \cos \theta), \\
\vec{\sigma} & =\left(\sigma_{x}, \sigma_{y}, \sigma_{z}\right) .
\end{aligned}
$$

Here, the Pauli matrices $\sigma_{i}$ are in the basis of $|101\rangle$ and $|110\rangle$.

A similar analysis can be performed for the effective $V$-type three-level system, i.e., the subspace spanned by $|111\rangle,|210\rangle$, and $|201\rangle$. Introducing the new basis states

$$
\left|B^{\prime}\right\rangle=\sin \frac{\theta}{2} e^{-i \phi}|210\rangle-\cos \frac{\theta}{2}|201\rangle,
$$

$$
\begin{aligned}
\left|D^{\prime}\right\rangle & =\cos \frac{\theta}{2}|210\rangle+\sin \frac{\theta}{2} e^{i \phi}|201\rangle, \\
\left|E^{\prime}\right\rangle & =|111\rangle,
\end{aligned}
$$

the Hamiltonian of this subspace can be written as

$$
H_{-}=\left(\Omega\left|B^{\prime}\right\rangle\left\langle E^{\prime}\right|+\text { H.c. }\right)-\delta\left|\mathrm{E}^{\prime}\right\rangle\left\langle\mathrm{E}^{\prime}\right| \text {. }
$$

Thus, time evolution until the gate time $t_{\text {gate }}$ will lead to both $\left|B^{\prime}\right\rangle$ and $\left|E^{\prime}\right\rangle$ acquiring a phase factor $-e^{i \gamma}$. However, $\left|B^{\prime}\right\rangle$ and $\left|D^{\prime}\right\rangle$ span the subspace of the states $|201\rangle$ and $|210\rangle$, neither of which is in the computational subspace, and thus will not be populated in the initial or final states of the gate. The only effect of the gate in the effective $V$-type three-level system is thus to bestow a phase factor $-e^{i \gamma}$ on $|111\rangle$.

\section{The family of three-qubit gates}

Summarizing the results from the analysis above, we see that the eight states in the computational subspace of the three qubits are affected as follows: $|101\rangle$ and $|110\rangle$ obey the time evolution given by Eq. (12), $|111\rangle$ will acquire a phase factor $-e^{i \gamma}$, and all the other states are unchanged. This is similar to the three-qubit Fredkin gate (controlledSWAP) $[2,19,101,102]$, which swaps the states of two target qubits conditioned on the state of a control qubit, i.e., $|101\rangle$ and $|110\rangle$ are swapped if the first qubit is the control qubit. Our gate, which we denote CCZs [see Fig. 1(c)], also implements a SWAP-like operation on the outer qubits $q_{1}$ and $q_{2}$, conditioned on the middle qubit $q_{0}$, but adds phase factors to $|101\rangle,|110\rangle$, and $|111\rangle$. The gate can be written as

$$
\begin{aligned}
\operatorname{CCZS}(\theta, \phi, \gamma)= & |0\rangle\left\langle\left. 0\right|_{0} \otimes \mathbb{I}_{1} \otimes \mathbb{I}_{2}\right. \\
& +|1\rangle\left\langle\left. 1\right|_{0} \otimes U_{\mathrm{CZS}}(\theta, \phi, \gamma),\right.
\end{aligned}
$$

where

$$
U_{\mathrm{CZS}}(\theta, \phi, \gamma)=\left[\begin{array}{cccc}
1 & 0 & 0 & 0 \\
0 & -e^{i \gamma} \sin ^{2}(\theta / 2)+\cos ^{2}(\theta / 2) & \frac{1}{2}\left(1+e^{i \gamma}\right) e^{-i \phi} \sin \theta & 0 \\
0 & \frac{1}{2}\left(1+e^{i \gamma}\right) e^{i \phi} \sin \theta & -e^{i \gamma} \cos ^{2}(\theta / 2)+\sin ^{2}(\theta / 2) & 0 \\
0 & 0 & 0 & -e^{i \gamma}
\end{array}\right]
$$

and the real parameters $\theta, \phi$, and $\gamma$ are set by the coupling strengths $\lambda_{1}, \lambda_{2}$ and the detuning $\delta$ according to the relations

$$
\gamma=\frac{\pi \delta}{\sqrt{4 \Omega^{2}+\delta^{2}}} \in(-\pi, \pi)
$$

$$
\Omega=\sqrt{\left|\lambda_{1}\right|^{2}+\left|\lambda_{2}\right|^{2}}
$$




\section{Examples of three-qubit gates}

It is illuminating to study a few of the simplest parameter choices for the CCZS gate. If we set $\lambda_{1}=\lambda, \lambda_{2}=0$, and $\delta=0$, we recover the two-qubit CZ gate acting on $q_{0}$ and $q_{1}$. In the same way, if instead $\lambda_{1}=0, \lambda_{2}=\lambda$, and $\delta=0$, we obtain the two-qubit CZ gate acting on $q_{0}$ and $q_{2}$. The gate time for these gates is $t_{\text {gate }}=\pi / \lambda$. We note that, for qubits that are close to harmonic oscillators, e.g., superconducting transmon qubits [103], the coupling strengths $\lambda_{j}=\sqrt{2} g_{j}$, where the $g_{j}$ are the coupling strengths for the $\left|1_{0} 0_{j}\right\rangle \leftrightarrow\left|0_{0} 1_{j}\right\rangle$ transitions. In this case, we can also write $t_{\text {gate }}=\pi / \sqrt{2} g$.

If we instead apply both these $\mathrm{CZ}$ gates simultaneously, i.e., $\lambda_{1}=\lambda_{2}=\lambda$ and $\delta=0$, we obtain $\operatorname{CCzS}(\theta=$ $\pi / 2, \phi=\pi, \gamma=0$ ), for which

$$
U_{\mathrm{CZS}}(\pi / 2, \pi, 0)=\left[\begin{array}{cccc}
1 & 0 & 0 & 0 \\
0 & 0 & -1 & 0 \\
0 & -1 & 0 & 0 \\
0 & 0 & 0 & -1
\end{array}\right] .
$$

The gate time for this gate is $t_{\text {gate }}=\pi / \sqrt{2} \lambda$, i.e., this threequbit gate is a factor $\sqrt{2}$ faster than the two-qubit $\mathrm{CZ}$ gates generated by the two interactions from which the CCZS gate is constructed.

We can set the phase $\phi$ by adjusting the relative phase of the coupling strengths $\lambda_{1}$ and $\lambda_{2}$. For $\lambda_{1}=\lambda, \lambda_{2}=-\lambda e^{i \phi}$, and $\delta=0$, the controlled part of the gate becomes

$$
U_{\mathrm{CZS}}(\pi / 2, \phi, 0)=\left[\begin{array}{cccc}
1 & 0 & 0 & 0 \\
0 & 0 & e^{-i \phi} & 0 \\
0 & e^{i \phi} & 0 & 0 \\
0 & 0 & 0 & -1
\end{array}\right] .
$$

The gate time remains $t_{\text {gate }}=\pi / \sqrt{2} \lambda$.

Further tuning can be achieved by changing the relative amplitudes of the coupling strengths $\lambda_{1}$ and $\lambda_{2}$. Setting $\lambda_{1}=\lambda, \lambda_{2}=-K \lambda e^{i \phi}$, and $\delta=0$, we have $\theta=2 \arctan K$ and the controlled part of the gate becomes

$$
U_{\mathrm{CZS}}(\theta, \phi, 0)=\left[\begin{array}{cccc}
1 & 0 & 0 & 0 \\
0 & \cos \theta & e^{-i \phi} \sin \theta & 0 \\
0 & e^{i \phi} \sin \theta & -\cos \theta & 0 \\
0 & 0 & 0 & -1
\end{array}\right] .
$$

The gate time becomes $t_{\text {gate }}=\pi / \sqrt{1+K^{2}} \lambda$. This is faster than the corresponding individual $\mathrm{CZ}$ gates, which would take $t_{\mathrm{gate}}=\pi / \lambda$ and $t_{\mathrm{gate}}=\pi / K \lambda$, respectively, on their own.

\section{Time-dependent parameters}

In the derivation of Eqs. (19)-(23), which constitute the main result of this section, we assumed for simplicity that the coupling strengths $\lambda_{1}, \lambda_{2}$ and the detuning $\delta$ were constants. However, in actual experiments, at least the coupling strengths $\lambda_{1}$ and $\lambda_{2}$ will need to be turned on and off, and this will not be done with a perfect step function. Fortunately, it is feasible to vary these parameters in time as long as they have the same time dependence. When the time dependence is the same, the eigenstates in Eqs. (2)-(4) remain time independent and the gate time remains easy to find. The principle is the same as for nonadiabatic holonomic gates [100,104-106], where adiabaticity is removed by constructing time-independent eigenstates. As Eq. (12) shows, we can thus construct arbitrary rotations in the space spanned by the states $|101\rangle$ and $|110\rangle$, where the angles $\theta, \phi$ are controlled by the relative strengths of the two $\mathrm{CZ}$ gates being applied simultaneously [see Eq. (21)].

\section{B. Decomposition into two-qubit gates}

The three-qubit CCZS gate entangles all three qubits. It can thus not be written as the simultaneous application of a two-qubit gate to two of the qubits and a single-qubit gate to the third qubit. Instead, decomposing the CCZS gate into single- and two-qubit gates requires the consecutive application of several such gates. Inspired by decompositions for the quantum-optical Fredkin gate [2,102], we find that the CCZS gate can be realized through the consecutive application of three two-qubit gates:

$$
\begin{aligned}
\operatorname{CCZS}(\theta, \phi, \gamma)= & \mathrm{XY}_{12}\left(\theta, \frac{\pi}{2}-\phi\right) \cdot \mathrm{CZ}_{01}(\gamma) \\
& \cdot \mathrm{XY}_{12}^{\dagger}\left(\theta, \frac{\pi}{2}-\phi\right),
\end{aligned}
$$

where

$$
\mathrm{XY}(\theta, \phi)=\left[\begin{array}{cccc}
1 & 0 & 0 & 0 \\
0 & \cos (\theta / 2) & i \sin (\theta / 2) e^{i \phi} & 0 \\
0 & i \sin (\theta / 2) e^{-i \phi} & \cos (\theta / 2) & 0 \\
0 & 0 & 0 & 1
\end{array}\right]
$$

and

$$
\mathrm{CZ}(\gamma)=\left[\begin{array}{cccc}
1 & 0 & 0 & 0 \\
0 & 1 & 0 & 0 \\
0 & 0 & 1 & 0 \\
0 & 0 & 0 & -e^{i \gamma}
\end{array}\right]
$$

Here, the two-qubit $\mathrm{XY}(\theta, \phi)$ gate $[107,108]$ is generated by an exchange-type interaction, e.g., $\mathrm{XY}(\pi, 0)=$ isWAP.

The decomposition in Eq. (27) is illustrated in Fig. 2. From that illustration, it becomes clear that this decomposition requires relabeling the qubits in a linear chain to work. For the case of a linear chain, the control qubit in the CCZS gate is the middle qubit, but in the decomposition given here, the control qubit must be one of the outer qubits. 

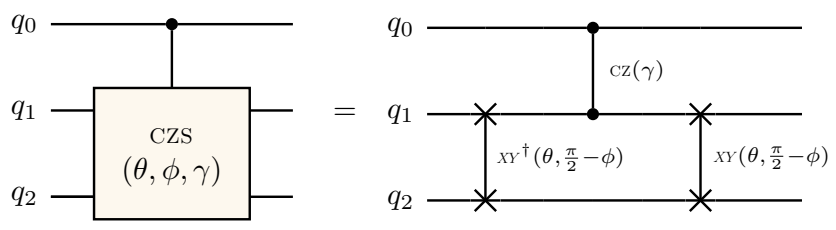

FIG. 2. Decomposition of the three-qubit CCZS gate into twoqubit gates. Note that, for the case of a linear chain [see Fig. 1(a)], the control qubit $q_{0}$ in the CCZS gate is the middle qubit, but the decomposition into two-qubit gates requires $q_{1}$ to be the middle qubit, since it has to interact with both $q_{0}$ and $q_{2}$.

\section{Constructing other three-qubit gates}

We now check how the CCZS gate family is related to some well-known three-qubit gates: the Fredkin (controlled-SWAP) [2,19,101,102], iFredkin (controlledisWAP) $[23,31,70]$, and Toffoli (controlled-controlled-NOT) $[2,109]$ gates. Just like the CCZS gate, these three-qubit gates can be written on the form of Eq. (19), but with $U_{\mathrm{CZS}}(\theta, \phi, \gamma)$ replaced by other controlled two-qubit unitary operations:

$$
\begin{aligned}
& U_{\text {Fredkin }}=\left[\begin{array}{llll}
1 & 0 & 0 & 0 \\
0 & 0 & 1 & 0 \\
0 & 1 & 0 & 0 \\
0 & 0 & 0 & 1
\end{array}\right], \\
& U_{\text {iFredkin }}=\left[\begin{array}{llll}
1 & 0 & 0 & 0 \\
0 & 0 & i & 0 \\
0 & i & 0 & 0 \\
0 & 0 & 0 & 1
\end{array}\right], \\
& U_{\text {Toffoli }}=\left[\begin{array}{llll}
1 & 0 & 0 & 0 \\
0 & 1 & 0 & 0 \\
0 & 0 & 0 & 1 \\
0 & 0 & 1 & 0
\end{array}\right] .
\end{aligned}
$$

Comparing with $U_{\mathrm{CZS}}(\theta, \phi, \gamma)$ in Eq. (20), it is clear that it never coincides with $U_{\text {Toffoli }}$ in Eq. (32), since the two off-diagonal elements in the lower right corner of $U_{\mathrm{CZS}}(\theta, \phi, \gamma)$ are zero for all values of $\theta, \phi$, and $\gamma$. Noting that a Toffoli gate is formed by sandwiching a controlledcontrolled- $Z$ (CCZ) gate [110] between two Hadamard gates on qubit 2 does not help. The controlled unitary of the $\mathrm{CCZ}$ gate is

$$
U_{\mathrm{CZ}}=\left[\begin{array}{cccc}
1 & 0 & 0 & 0 \\
0 & 1 & 0 & 0 \\
0 & 0 & 1 & 0 \\
0 & 0 & 0 & -1
\end{array}\right]
$$

To obtain the -1 in this matrix from $U_{\mathrm{CZS}}(\theta, \phi, \gamma)$ requires $\gamma=0$, which is easy, but we see from Eq. (26) that the two (a)
Fredkin

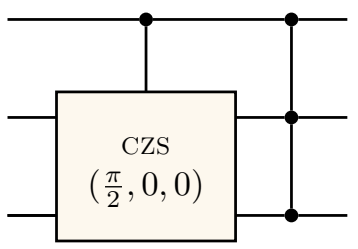

(b)

iFredkin

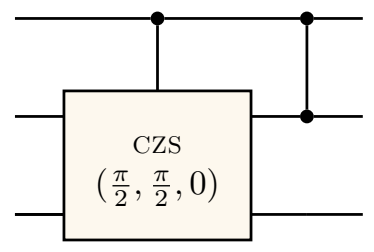

FIG. 3. Converting CCZS gates into other three-qubit gates. (a) Constructing a Fredkin gate with a $\operatorname{CCZS}(\pi / 2,0,0)$ gate and a $\mathrm{CCZ}$ gate. (b) Constructing an iFredkin gate from a $\operatorname{CCZS}(\pi / 2, \pi / 2,0)$ gate and a CZ gate.

middle diagonal elements in $U_{\mathrm{CZS}}(\theta, \phi, \gamma)$ then always will have opposite signs, which does not match the $\mathrm{CCZ}$ gate. Indeed, for $\theta=0$, we simply have the two-qubit $\mathrm{CZ}$ gate acting on qubits 0 and 1 .

The Fredkin gate in Eq. (30) also cannot be directly implemented by the CCZS gate. For the 1 in the lower right corner of $U_{\text {Fredkin }}$ to match $U_{\mathrm{CZS}}(\theta, \phi, \gamma), \gamma=$ $\pm \pi$ is necessary, but then all off-diagonal elements in $U_{\mathrm{CZS}}(\theta, \phi, \gamma)$ become zero. However, we note that the Fredkin gate can be constructed by combining a CCZ gate and $\operatorname{cczs}(\pi / 2,0,0)$ (i.e., $\lambda_{1}=-\lambda_{2}$ and $\delta=0$ ), as shown in Fig. 3(a). Since an implementation of the Fredkin gate using only single- and two-qubit gates requires at least five two-qubit gates [19], and a CCZ gate can be implemented using three two-qubit gates [110], the construction with the CCZS gate (which on its own is at least as fast as a two-qubit gate) constitutes an improvement.

Using exactly the same reasoning as for the Fredkin gate in the preceding paragraph, we see that the iFredkin gate in Eq. (31) also cannot be directly implemented by the CCZS gate. However, it is sufficient to add a single twoqubit $\mathrm{CZ}$ gate after $\operatorname{CCZS}(\pi / 2, \pi / 2,0)$ to fix this, as shown in Fig. 3(b). Since implementing the iFredkin gate using only single- and two-qubit gates requires at least four twoqubit gates, while the CCZS gate is at least as fast as a two-qubit gate, our construction at least halves the time required for the iFredkin gate, which is a natural operation in, e.g., simulations of the Fermi-Hubbard model [70].

\section{Rapid creation of large entangled states}

Having demonstrated that the CCZS gate is fast and that it entangles three qubits, we now show how this gate and its generalization to more than three qubits can be applied to rapidly generate some particular large entangled states. The ability to create entanglement [111] is crucial for both quantum information processing $[2,112,113]$ and quantum communication [114]. Lately, the creation of entanglement between (several) tens of qubits has been used to demonstrate the capabilities of quantum processors on multiple 
(a)

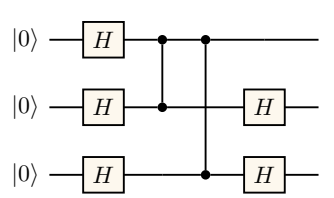

(b)

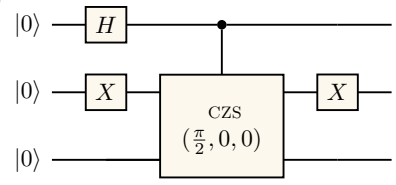

FIG. 4. Quantum circuits for generating the three-qubit GHZ state $(|000\rangle+|111\rangle) / \sqrt{2}$. (a) A circuit using single-qubit gates and two-qubit CZ gates. (b) A circuit using single-qubit gates and one CCZS gate.

platforms: superconducting circuits [15,115-118], photonic systems [115], ion traps [62,119], and neutral atoms [120].

\section{Greenberger-Horne-Zeilinger states}

GHZ states [71,72] are entangled states of $N$ qubits on the form

$$
\left|\Psi_{\mathrm{GHZ}}\right\rangle=\frac{1}{\sqrt{2}}\left(|0\rangle^{\otimes N}+|1\rangle^{\otimes N}\right) .
$$

To generate a GHZ state with $N=3$ qubits using only single- and two-qubit gates requires at least two twoqubit gates, e.g., two controlled-NOT (CNOT) gates, two isWAP gates [121], or two CZ gates [see Fig. 4(a)]. However, this state can also be generated using only a single CCZS gate and a few single-qubit gates, using the circuit shown in Fig. 4(b). Starting from $|000\rangle$, applying singlequbit Hadamard and $X$ gates to the first and second qubit, respectively, creates the state

$$
|\psi\rangle=\frac{1}{\sqrt{2}}(|0\rangle+|1\rangle)|1\rangle|0\rangle
$$

Then, applying $\operatorname{CCZs}(\theta=\pi / 2, \phi=0, \gamma=0)$, which is achieved for $\lambda_{1}=-\lambda_{2}$ and $\delta=0$, results in a controlled SWAP of the second and third qubits [see Eq. (25)], yielding the state

$$
\operatorname{CCZS}\left(\frac{\pi}{2}, 0,0\right)|\psi\rangle=\frac{1}{\sqrt{2}}(|010\rangle+|101\rangle),
$$

which is transformed to the three-qubit GHZ state by applying an $X$ gate on the second qubit. We remark that the phase acquired by the doubly excited state of the second and third qubits does not affect the state $|\psi\rangle$ that we apply the CCZS gate to.

Since $\operatorname{CCZS}(\pi / 2,0,0)$ is $\sqrt{2}$ faster than the CZ gates that can be implemented with the interactions from which it is constructed (see Sec. 3), the circuit with the CCZS gate in Fig. 4(b) generates the three-qubit GHZ state $2 \sqrt{2}$ times faster than the circuit with the two CZ gates in Fig. 4(a), provided that the time required for single-qubit gates is negligible. If one instead uses the circuit with two isWAP

gates [121], the circuit with the CCZS gate is twice as fast, since the isWAP gate is $\sqrt{2}$ faster than a $\mathrm{CZ}$ gate in the setups we consider here (the coupling between $|11\rangle$ and $|02\rangle$ is $\sqrt{2}$ stronger than the coupling between $|01\rangle$ and $|10\rangle$ for weakly anharmonic qubits, but the iswAP gate only requires half the oscillation between states that the $\mathrm{CZ}$ gate does; see, e.g., Ref. [98]).

\section{Dicke states}

Another class of entangled states is the $\mathrm{W}$ states

$\left|\Psi_{W}\right\rangle=\frac{1}{\sqrt{N}}(|100 \cdots 0\rangle+|010 \cdots 0\rangle+\cdots+|000 \cdots 1\rangle)$,

which cannot be converted into GHZ states by local operations and classical communication [122]. The $\mathrm{W}$ states are in turn a subset $\left|D_{N}^{1}\right\rangle$ of the symmetric Dicke states $\left|D_{N}^{k}\right\rangle$ [73-75], which are equally weighted superpositions of all permutations of $N$-qubit states with $k$ excitations:

$$
\left|D_{N}^{k}\right\rangle=\frac{1}{\sqrt{\left(\begin{array}{c}
N \\
k
\end{array}\right)}} \mathcal{S}\left[|0\rangle^{\otimes(N-k)} \otimes|1\rangle^{\otimes k}\right]
$$

with $\mathcal{S}$ the symmetrization operator.

Dicke states have important applications in quantum metrology $[123,124]$ and quantum networks [125-128]. Recently, it has also been shown that, for combinatorial optimization problems, symmetric Dicke states representing a superposition of all feasible solutions can give advantages when used as the initial state in the quantum alternating operator ansatz $[129,130]$.

The Dicke states arise naturally when $N$ identical atoms are collectively coupled to a harmonic mode [74,131]. However, since the photon or phonon number of the harmonic mode is difficult to control, alternative protocols for Dicke-state generation have been proposed [132-136]. For deterministic preparation of a symmetric Dicke state on a quantum computer, using a sequence of single- and two-qubit gates, it has been shown that constructing $\left|D_{N}^{k}\right\rangle$ requires a quantum circuit with depth $\mathcal{O}(N)$ containing at least $\mathcal{O}(k N)$ gates [137-139].

In this subsection, we show how to rapidly create large symmetric Dicke states by generalizing the interaction underpinning the CCZS gate to more qubits. As a concrete example, we show that we can create the state $\left|D_{5}^{3}\right\rangle$ using only two rounds of simultaneous $\mathrm{CZ}$ gates and two singlequbit operations, while the quantum circuit in Ref. [138] for creating the same state includes five three-qubit gates and 21 two-qubit gates applied sequentially.

a. Hamiltonian and dynamics. We consider a system composed of a central qubit 0 and its $N$ nearest neighbors $\{j\}$ (the three-qubit system in Fig. 1 had $\{j\}=\{1,2\}$ ). For each 
qubit $i$, we take into account the three lowest energy levels $\left|0_{i}\right\rangle,\left|1_{i}\right\rangle$, and $\left|2_{i}\right\rangle$, with energies $0, \omega_{i}$, and $2 \omega_{i}+\alpha_{i}$, respectively, where $\alpha_{i}$ is the anharmonicity. In this system, a CZ gate between qubit 0 and one of its neighbors $j$ can be applied by activating the $\left|2_{0} 0_{j}\right\rangle \leftrightarrow\left|1_{0} 1_{j}\right\rangle$ transition. Assuming that these transitions are resonant, the system Hamiltonian with the transitions switched on is, in the interaction picture,

$$
H=\sigma_{0}^{21} \sum_{j=1}^{N} \lambda_{j} \sigma_{j}^{01}+\text { H.c. }
$$

where $\sigma_{i}^{n m}=|n\rangle\left\langle\left. m\right|_{i}\right.$ and $\lambda_{j}$ is the interaction strength for the $\left|2_{0} 0_{j}\right\rangle \leftrightarrow\left|1_{0} 1_{j}\right\rangle$ transition.

For simplicity, we assume that all interactions are equally strong: $\lambda_{j} \equiv \lambda$. We can then introduce the collective spin operator $J^{-}=\sum_{j=1}^{N} \sigma_{j}^{01}$ and rewrite Eq. (39) as

$$
H=\lambda \sigma_{0}^{21} J^{-}+\text {H.c., }
$$

which is reminiscent of the Tavis-Cummings (Dicke) model [140,141], where a harmonic oscillator couples to $N$ identical atoms. Because of the anharmonicity of qubit 0 , the models are equivalent in the limit where the harmonic oscillator only hosts a single photon.

The neighboring qubits 1 to $N$ are symmetric under permutation and can thus be described by the Dicke states in Eq. (38). In this basis, the matrix elements of the operator $J^{+}=\left(J^{-}\right)^{\dagger}$ are [142]

$$
\begin{aligned}
\left\langle D_{N}^{k+1}\left|J^{+}\right| D_{N}^{k}\right\rangle & =(N-k) \sqrt{\frac{\left(\begin{array}{c}
N \\
k
\end{array}\right)}{\left(\begin{array}{c}
N \\
k+1
\end{array}\right)}} \\
& =\sqrt{(N-k)(k+1)} \\
& \equiv G_{N}^{k} .
\end{aligned}
$$

We can thus interpret the second term in Eq. (40) as qubit 0 being de-excited from $\left|2_{0}\right\rangle$ to $\left|1_{0}\right\rangle$ while the state of the neighboring qubits changes from $\left|D_{N}^{k}\right\rangle$ to $\left|D_{N}^{k+1}\right\rangle$. The total excitation number $k+2$ is conserved.

Since subspaces with different numbers of excitations are decoupled, we can limit ourselves to the subspace with $k+2$ excitations, which is spanned by the basis $\left|1_{0}\right\rangle\left|D_{N}^{k+1}\right\rangle,\left|2_{0}\right\rangle\left|D_{N}^{k}\right\rangle$. Using Eqs. (40)-(41), we see that the dynamics in this subspace is generated by

$$
\exp \left(-i t \lambda G_{N}^{k} \tilde{\sigma}_{x}\right)=\cos \left(t \lambda G_{N}^{k}\right)-i \sin \left(t \lambda G_{N}^{k}\right) \tilde{\sigma}_{x}
$$

where $\tilde{\sigma}_{x}$ is defined in the basis of the subspace.

$b$. Generalizing the CCZS gate to more than three qubits. We can now understand how the CCZS gate generalizes to more qubits. For $N=2$ neighboring qubits, starting in the computational subspace, i.e., with qubit 0 in state $\left|1_{0}\right\rangle$, we can always find a time $t$ when the occupation of $\left|2_{0}\right\rangle$ is zero, since $G_{2}^{1}=G_{2}^{0}=\sqrt{2}$. This is the case analyzed in the preceding subsections. For $N>2$, the coefficients $G_{N}^{k}$ are not equal or commensurate, making it impossible to confine the central qubit to the computational subspace and create an $(N+1)$-qubit gate according to the same principle as the CCZS gate. What could be done is to apply a singlequtrit operation on qubit 0 that takes $\left|0_{0}\right\rangle$ to $\left|1_{0}\right\rangle$ and $\left|1_{0}\right\rangle$ to $\left|2_{0}\right\rangle$, let the system evolve for some time $t$ according to Eq. (42), and then apply the inverse of the single-qutrit operation to qubit 0 to bring it back to the computational subspace. While this would be an $(N+1)$-qubit gate, it appears too complicated to find immediate applications.

c. Creating a five-qubit Dicke state. Instead of constructing a general $(N+1)$-qubit gate, we therefore focus on preparing symmetric Dicke states by starting in a specific subspace. As an illustrative example, we consider the case $N=4$ and the target state

$$
\left|D_{5}^{3}\right\rangle=\sqrt{\frac{3}{5}}\left|1_{0}\right\rangle\left|D_{4}^{2}\right\rangle+\sqrt{\frac{2}{5}}\left|0_{0}\right\rangle\left|D_{4}^{3}\right\rangle,
$$

which was also used as an example in Ref. [138].

First, we note that, since $\left|D_{4}^{3}\right\rangle$ can be created by applying $X$ gates to all qubits in the state $\left|D_{4}^{1}\right\rangle$, and the state $\left|D_{4}^{2}\right\rangle$ is unchanged by those gates, the problem reduces to preparing the state $\sqrt{(3 / 5)}\left|1_{0}\right\rangle\left|D_{4}^{2}\right\rangle+\sqrt{(2 / 5)}\left|0_{0}\right\rangle\left|D_{4}^{1}\right\rangle$. The procedure for doing so is illustrated in Fig. 5. We first explain how to obtain $\left|D_{4}^{2}\right\rangle$.

1. First, we prepare the initial state $|20000\rangle=\left|2_{0}\right\rangle\left|D_{4}^{0}\right\rangle$ by single-qutrit operations on qubit 0 . This puts the system in the subspace $k=0$ spanned by $\left|1_{0}\right\rangle\left|D_{4}^{1}\right\rangle$ and $\left|2_{0}\right\rangle\left|D_{4}^{0}\right\rangle$. Turning on the interaction and letting the system evolve for a time $t=\pi /(4 \lambda), 4$ times faster than a two-qubit $\mathrm{CZ}$ gate, we arrive at $\left|1_{0}\right\rangle\left|D_{4}^{1}\right\rangle$. We remark that if we have the system tuned to have different interaction strengths $\lambda_{j}$ like in tripod systems $[143,144]$, we can create arbitrarily weighted superpositions of the $N$-qubit states with one excitation instead of the symmetric superposition that is the symmetric Dicke state.

2. Next, we flip qubit 0 to $\left|2_{0}\right\rangle$ such that the system state becomes $\left|2_{0}\right\rangle\left|D_{4}^{1}\right\rangle$. This puts the system in the $k=1$ subspace spanned by $\left|1_{0}\right\rangle\left|D_{4}^{2}\right\rangle$ and $\left|2_{0}\right\rangle\left|D_{4}^{1}\right\rangle$. Turning on the interaction again for a time $t=$ $\pi /(2 \sqrt{6} \lambda)$, we arrive at $\left|1_{0}\right\rangle\left|D_{4}^{2}\right\rangle$.

To create the superposition state in Eq. (43), we carry out step 1 as above. Then we rotate qubit 0 to the superposition state $\sqrt{(2 / 5)}\left|0_{0}\right\rangle+\sqrt{(3 / 5)}\left|1_{0}\right\rangle$ and flip $\left|1_{0}\right\rangle$ to $\left|2_{0}\right\rangle$, yielding the system state $\left[\sqrt{(2 / 5)}\left|0_{0}\right\rangle+\sqrt{(3 / 5)}\left|2_{0}\right\rangle\right]\left|D_{4}^{1}\right\rangle$. Turning on the interaction as in step 2 above, the part of 


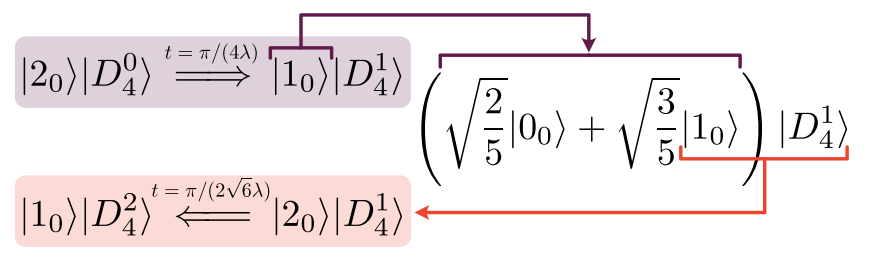

FIG. 5. The steps for preparing the state $(\sqrt{3 / 5})\left|1_{0}\right\rangle\left|D_{4}^{2}\right\rangle+$ $(\sqrt{2 / 5})\left|0_{0}\right\rangle\left|D_{4}^{1}\right\rangle$ using the generalization of the CCZS gate to a five-qubit system.

the superposition containing $\left|0_{0}\right\rangle$ is decoupled from the dynamics, while the part containing $\left|2_{0}\right\rangle$ reaches $\left|1_{0}\right\rangle\left|D_{4}^{2}\right\rangle$ as before. Finally, applying $X$ gates to the four neighboring qubits yields the state in Eq. (43).

In total, our scheme requires seven single-qubit operations (four of them simultaneous) and two applications of the interaction that yields $\mathrm{CZ}$ gates. The total time spent on these $\mathrm{CZ}$ interactions is less than that of single two-qubit CZ gates. This fast creation of the entangled state in Eq. (43) should be contrasted with the quantum circuit for the same task given in Ref. [138], which contained five three-qubit gates and 21 two-qubit gates, applied sequentially.

d. Rapid creation of a large $W$ state. The size of the Dicke state that can be efficiently prepared is determined by the number of neighboring qubits to which the central qubit is coupled. In general, scaling up arbitrary Dicke states is hard [145]. However, in our scheme, large $W$ states are easy to construct even with limited connectivity, e.g., in a square grid of qubits, as shown in Fig. 6. The method outlined there is straightforward to adapt for other connectivities.

\section{E. Experimental feasibility}

To determine how well the CCZS gate is likely to work in actual experiments, we now turn to simulating two specific possible experimental implementations of the gate. We consider two gate schemes commonly used to perform $\mathrm{CZ}$ gates in superconducting circuits by turning on and off an interaction between the two-qubit states $|11\rangle$ and $|02\rangle$ or $|20\rangle$. Similar schemes used for $\mathrm{CZ}$ gates on other quantum-computing platforms should be equally feasible for realizing the CCZS gate or variants thereof. For example, CZ gates can be implemented with trapped ions or Rydberg atoms by exciting the qubits from the excited state $|1\rangle$ to a higher excited state $|r\rangle[61,63,65]$. The two ions or atoms will experience a Rydberg interaction if and only if both of them are in $|r\rangle$. This is known as Rydberg blockade and can be used to impart a phase shift on the state $|11\rangle$ while leaving all other states unchanged. In a threequbit chain, the application of simultaneous $\mathrm{CZ}$ gates will change the Rydberg interaction experienced by the middle qubit, leading to a three-qubit gate [28].

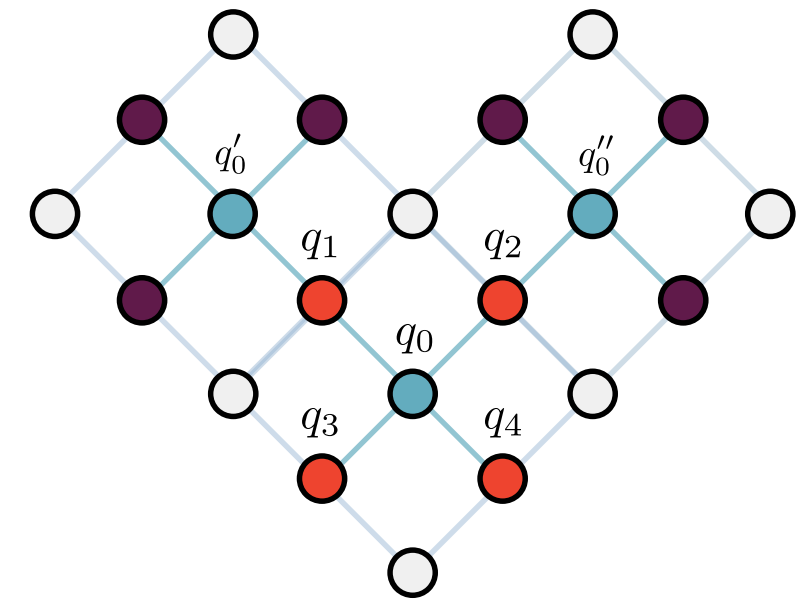

FIG. 6. Creating large $\mathrm{W}$ states rapidly on a square grid of qubits. We first prepare qubit $0\left(q_{0}\right)$ in its second excited state and carry out the rest of step 1 in Sec. II D 2 c to create $\left|D_{4}^{1}\right\rangle$ on the neighboring qubits (red circles). Next, we swap each of the neighboring qubits with the qubit next to them that is farthest from the center qubit (blue circles except $q_{0}$ ). Flipping the $|1\rangle$ part of the state for these new center qubits to $|2\rangle$ and having them interact with their nearest neighbors creates $\left|D_{4}^{1}\right\rangle$ on those neighbors (purple and red circles). For brevity, the new cells starting from $q_{3}$ and $q_{4}$ are not shown. The result after these steps (two rounds of single or simultaneous single-qubit operations, three rounds of simultaneous two-qubit gates) is the 16-qubit W state $\left|D_{16}^{1}\right\rangle$.

In the first scheme for superconducting qubits, the two outer qubits in the three-qubit chain are tunable [89$91,146]$. To activate the gate, they are tuned such that the $|110\rangle$ and $|101\rangle$ states both become resonant with the $|200\rangle$ state. In some implementations, tunable couplers between the qubits are also adjusted to further control the coupling [92-94]. In all these cases, the interaction strengths $\lambda_{1}$ and $\lambda_{2}$ will be limited to being in phase, i.e., $\phi=\pi$ [see Eq. (21)]. In the cases without tunable couplers, the parameter $\theta$ [see Eq. (21)] is fixed by the coupling strengths in the hardware and cannot be tuned in situ.

In the second scheme, the neighboring qubits in the chain are connected via a tunable coupler, which itself is a qubit [95-98]. To activate a $\mathrm{CZ}$ gate, the coupler, which is detuned from the qubits it is connected to, is parametrically modulated with a modulation frequency close to the difference in frequency between the states $|11\rangle$ and $|02\rangle$ or $|20\rangle$. In this case, the interaction strengths $\lambda_{1}$ and $\lambda_{2}$ are determined by the phase and amplitude of the modulation of the coupler; they can thus be tuned over a wide range to implement different parameters for the CCZS gate. We note that a $\mathrm{CZ}$ gate can also be implemented in a similar fashion between a fixed-frequency qubit and a parametrically modulated qubit $[147,148]$, but we do not simulate that case here. 
To characterize the performance of the gates, we calculate the average gate fidelity $[149,150]$

$$
\begin{aligned}
F & =\int d|\psi\rangle\left\langle\psi\left|U^{\dagger} M\right| \psi\right\rangle\left\langle\psi\left|M^{\dagger} U\right| \psi\right\rangle \\
& =\frac{\left|\operatorname{Tr}\left(M U^{\dagger}\right)\right|^{2}+\operatorname{Tr}\left(M^{\dagger} M\right)}{n(n+1)},
\end{aligned}
$$

where $U$ is the ideal gate operation that we wish to implement, $M$ is the gate operation that we actually implement, and $n$ is the dimension of the computational subspace $\left(n=2^{2}=4\right.$ for the $\mathrm{CZ}$ gates and $n=2^{3}=8$ for the CCZS gate). Note that in all numerical simulations below, calculations are done by solving the Schrödinger equation in QuTiP $[151,152]$ with three or more levels per qubit. The results of the simulations are then truncated to the computational subspace in such a way that $M$ includes effects of any leakage to states outside the computational subspace, which will lead to $\operatorname{Tr}\left(M^{\dagger} M\right)<n$. The aim of the numerical simulations is to show that high-fidelity three-qubit CCZS gates can be obtained in a straightforward way, without optimizing pulse shapes, etc., compared to the constituent two-qubit gates.

\section{Tunable qubits}

We first consider the setup with two tunable qubits 1 and 2 on each side of the fixed-frequency qubit 0 , as shown in Fig. 7(a). To test the performance under realistic conditions, we use parameters close to the experiment in Ref. [90]. The Hamiltonian for the setup is

$$
\begin{aligned}
H= & \omega_{0} a_{0}^{\dagger} a_{0}+\frac{\alpha_{0}}{2} a_{0}^{\dagger} a_{0}\left(a_{0}^{\dagger} a_{0}-1\right) \\
& +\sum_{j=1,2}\left[\omega_{j}(t) a_{j}^{\dagger} a_{j}+\frac{\alpha_{j}}{2} a_{j}^{\dagger} a_{j}\left(a_{j}^{\dagger} a_{j}-1\right)\right] \\
& +\sum_{j=1,2} \frac{\lambda_{j}}{\sqrt{2}}\left(a_{0}^{\dagger}+a_{0}\right)\left(a_{j}^{\dagger}+a_{j}\right),
\end{aligned}
$$

where $a_{j}\left(a_{j}^{\dagger}\right)$ is the annihilation (creation) operator of qubit $j$. Before the gate is turned on, the qubit energies are

$$
\left[\omega_{0}, \omega_{1}, \omega_{2}\right]=2 \pi \times[5.202,5.708,4.350] \mathrm{GHz} .
$$

For qubits 0 and 1 , these are also their maximum energies; for qubit 2 , the maximum energy is set to $2 \pi \times 4.927 \mathrm{GHz}$. The qubit anharmonicities are

$$
\left[\alpha_{0}, \alpha_{1}, \alpha_{2}\right]=-2 \pi \times[275.2,261.1,277.3] \mathrm{MHz}
$$

and the couplings between the qubits are

$$
\lambda_{1}=\lambda_{2}=2 \pi \times \sqrt{2} \times 3.8 \mathrm{MHz} .
$$
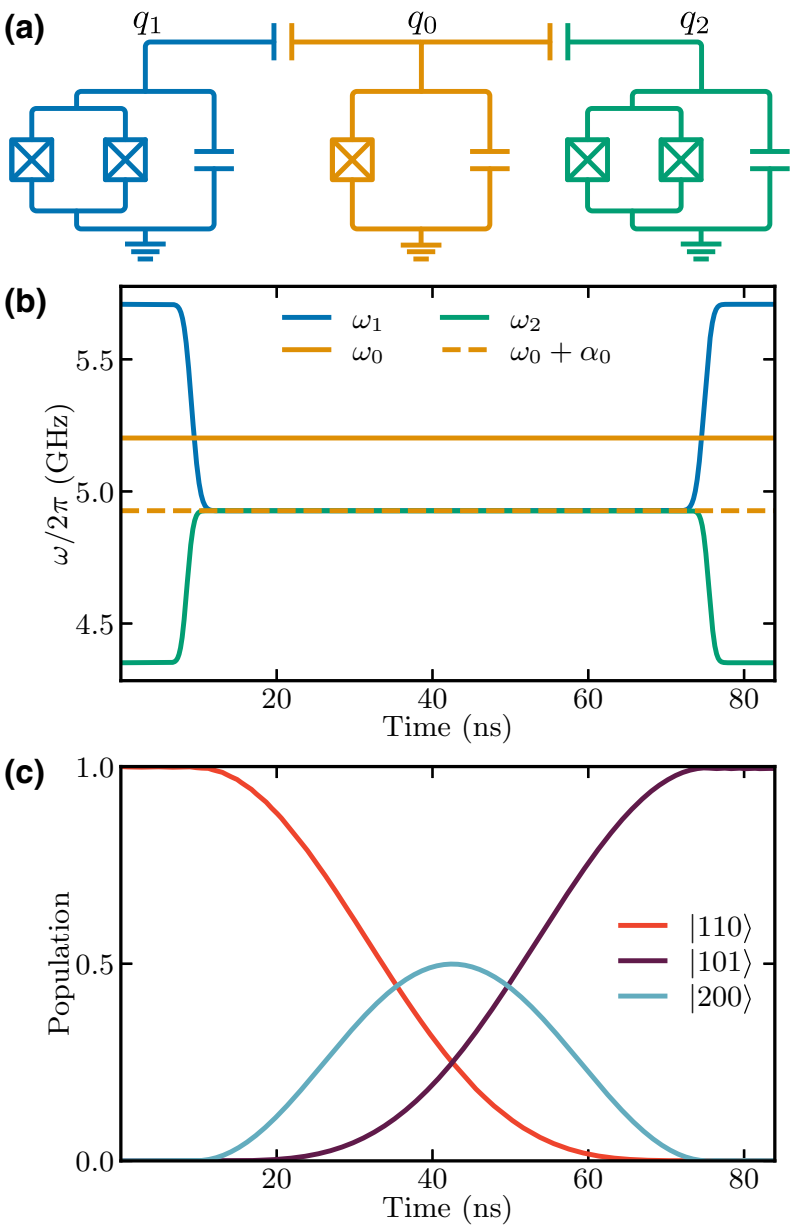

FIG. 7. Implementing a CCZS gate with tunable qubits. (a) A sketch of the setup, with qubit numbering following the convention established in Fig. 1(a). The superconducting qubits are transmon qubits [103], which are nonlinear $L C$ oscillators where the nonlinear inductances are provided by Josephson junctions (boxes with crosses in the sketch). When two Josephson junctions are combined in a loop [a superconducting quantum interference device (SQUID)], the effective inductance, and thus the qubit frequency, can be tuned by controlling the magnetic flux through the loop. (b) The tuning of the qubit energies used to implement a high-fidelity $\operatorname{CCZS}(\pi / 2, \pi, 0)$ gate. (c) Population of the states $|110\rangle,|200\rangle$, and $|101\rangle$ during the $\operatorname{CCZS}(\pi / 2, \pi, 0)$ gate when the initial state is $|110\rangle$. As Eq. (24) shows, the main effect of this gate is to swap the population between qubits 1 and 2 if qubit 0 is in its excited state.

The factor $\sqrt{2}$ appears in Eq. (48) since we use $\lambda_{1}$ and $\lambda_{2}$ to denote the coupling strengths for $|11\rangle \leftrightarrow|20\rangle$ transitions instead of the coupling strengths for $|10\rangle \leftrightarrow|01\rangle$ transitions, which are the parameters that are actually given as input to the simulation.

To activate the gate, energies $\omega_{1}$ and $\omega_{2}$ of qubits 1 and 2 are tuned into resonance with $\omega_{0}+\alpha_{0}$, as shown in Fig. 7(b). To account for the finite response time of the drive line, the pulse used for tuning the qubit energies is 
the convolution of a rectangular pulse of length $t_{\text {gate }}$ (the gate time) and a Gaussian pulse centered in the middle of the rectangular pulse with standard deviation $\sigma=1 \mathrm{~ns}$.

We first check that we can tune up $\mathrm{CZ}$ gates between qubits 1 or 2 and qubit 0 by tuning just one of qubits 1 and 2 to the relevant resonance. We find that we can achieve $F>99.99 \%$ for the $\mathrm{CZ}$ gate between qubits 0 and 2 with a gate time $t_{\text {gate, }} \mathrm{CZ}_{02}=93.0 \mathrm{~ns}$ and, similarly, $F=99.82 \%$ for the $\mathrm{CZ}$ gate between qubits 0 and 1 with a gate time $t_{\text {gate, }, \mathrm{CZ}_{01}}=94.0 \mathrm{~ns}$.

We then tune up the $\operatorname{CCZS}(\pi / 2, \pi, 0)$ gate [see Eq. (24)] by reducing the gate time and synchronizing the tuning of both qubits 1 and 2 to the resonance for the gate. Note that $\theta=\pi / 2$ is set by the fixed coupling strengths $\lambda_{1}=\lambda_{2}$ and cannot be changed. The best gate fidelity we find is $F=99.46 \%$ for the gate time $t_{\text {gate, } \mathrm{CCZS}}=66.8 \mathrm{~ns} \approx$ $t_{\text {gate, CZ }} / \sqrt{2}$ [see Figs. 7(b) and 7(c)]. In all simulations, we used four energy levels per qubit, so any effects of leakage outside the computational subspace are included in these results. However, note that the fidelity values for both the CCZS and CZ gates here are calculated without including any effects of decoherence.

We note that state-of-the-art nanofabrication techniques will still yield some deviations from the design values for the coupling strengths $\lambda_{1}$ and $\lambda_{2}$. These deviations can be on the order of $10 \%$ [90]. To quantify the impact of such deviations, we optimize the $\operatorname{CCZS}(\pi / 2, \pi, 0)$ gate with $\lambda_{2}=1.1 \lambda_{1}$ (instead of $\lambda_{2}=\lambda_{1}$ that we used above) and find a gate fidelity of $99.28 \%$. However, for the $\operatorname{CCZS}\left(2 \arctan \lambda_{2} / \lambda_{1}, \pi, 0\right)$ gate, which is the most suited to unequal couplings, we find a fidelity of $99.48 \%$. It is thus still possible to implement a high-fidelity CCZS gate with coupling strengths deviating from design values; the most high-fidelity gate will just have a slightly different $\theta$ than intended. For the $10 \%$ difference in coupling strength considered here, the optimal $\theta$ changed by $2 \arctan 1.1-\pi / 2=0.095 \mathrm{rad}=5.5^{\circ}$.

We attribute the deviation from $100 \%$ gate fidelity for the three-qubit gate in Fig. 7 to a combination of factors. One is imperfections arising when tuning qubits 1 and 2 in and out of the resonance. During that time, qubit 1 crosses the frequency of qubit 0 , which may cause leakage by briefly activating the $|01\rangle \leftrightarrow|10\rangle$ transition for these qubits instead of the desired $|11\rangle \leftrightarrow|20\rangle$ transition. We note that qubit 2 , which is below qubit 0 in frequency, does not have the same potential problem; this may explain why the $\mathrm{CZ}_{02}$ gate has higher fidelity than the $\mathrm{CZ}_{01}$ gate. Data from simulations with the initial state being $|100\rangle,|010\rangle$, and $|001\rangle$, shown in Appendix B, support this explanation. When the initial state is $|100\rangle(|010\rangle)$, we observe an increase of the $|010\rangle(|100\rangle)$ population of about $4 \times 10^{-3}$ occurring at $t \approx 10 \mathrm{~ns}$, consistent with a brief activation of the $|01\rangle \leftrightarrow|10\rangle$ transition between qubits 0 and 1 . When the initial state is $|001\rangle$, any population transfers are instead at least one order of magnitude lower.
Furthermore, tuning qubits 1 and 2 from different frequencies into the resonance appears to affect the parameter $\phi$, making it deviate from $\pi$ and thus lowering the gate fidelity. To improve the tuning of the qubit energies, one could try methods developed for nonadiabatic holonomic gates [153]. In that paper, it is shown that, while $\lambda_{1}$ and $\lambda_{2}$ should have the same time dependence, there is some freedom to choose the time dependence of $\delta$. This freedom can then be used to implement various optimal-control methods to improve the robustness of the gate.

An additional source of error may be that the states $|001\rangle,|100\rangle$, and $|010\rangle$ form a $\Lambda$ system during the gate operation, with $|001\rangle$ and $|010\rangle$ having the same energy. Although $|100\rangle$ is detuned from the other two states by the anharmonicity $\alpha_{0}$, there will still be a small effective coupling between $|001\rangle$ and $|010\rangle$ that can contribute to lowering the gate fidelity. This effect can be reduced by increasing the anharmonicity.

To further put the time gained by performing the threequbit gate in perspective, we show in Fig. 8 the decomposition of the $\operatorname{CCZS}(\pi / 2, \pi, 0)$ gate into single-qubit gates and two-qubit CZ gates between the middle qubit and its neighbors. Note that this is different from the decomposition in Fig. 2, which assumes access to a parametrized XY gate in addition to the $\mathrm{CZ}$ gates we have here. From the decomposition in Fig. 8, we see that five sequential $\mathrm{CZ}$ gates would be needed to implement this three-qubit gate in the setup at hand. Even if we assume that single-qubit gates take negligible time compared to two-qubit gates, this still means that we gain more than a factor 7 in gate time by implementing the three-qubit gate using our scheme.

\section{Tunable couplers}

a. Setup and operation. We next consider the setup with tunable couplers as shown in Fig. 9. This setup is modeled with the Hamiltonian

$$
\begin{aligned}
H= & \sum_{i=0,1,2}\left[\omega_{i} a_{i}^{\dagger} a_{i}+\frac{\alpha_{i}}{2} a_{i}^{\dagger} a_{i}\left(a_{i}^{\dagger} a_{i}-1\right)\right] \\
& +\sum_{j=1,2}\left[\omega_{c_{j}}(t) b_{j}^{\dagger} b_{j}+\frac{\alpha_{c_{j}}}{2} b_{j}^{\dagger} b_{j}\left(b_{j}^{\dagger} b_{j}-1\right)\right] \\
& +\sum_{\substack{i=0,1,2 \\
j=1,2}} g_{i j}\left(a_{i}^{\dagger}+a_{i}\right)\left(b_{j}^{\dagger}+b_{j}\right),
\end{aligned}
$$

where $a_{i}$ and $a_{i}^{\dagger}\left(b_{j}\right.$ and $\left.b_{j}^{\dagger}\right)$ are the annihilation and creation operators, respectively, of qubit $i$ (coupler $j), \omega_{i}\left(\omega_{c_{j}}\right)$ is its transition frequency, $\alpha_{i}\left(\alpha_{c_{j}}\right)$ its anharmonicity, and $g_{i j}$ is the strength of the capacitive coupling between qubit $i$ and coupler $j$. We use parameter values similar to recent updates of the design in Ref. [97]. These values, which are kept fixed throughout all simulations, are given in Table I. 


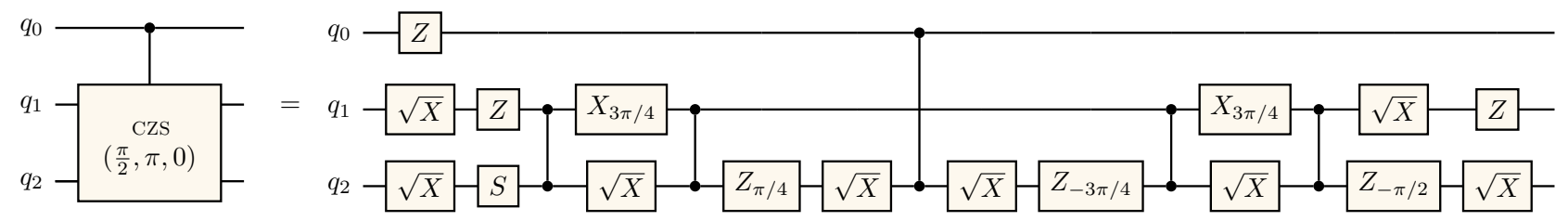

FIG. 8. Decomposition of the $\operatorname{CCZS}(\pi / 2, \pi, 0)$ gate into single-qubit and CZ gates, obtained using Qiskit [154]. For the single-qubit gates, we use the notation $S=\sqrt{Z}=Z_{\pi / 2}$ and $\sqrt{X}=X_{\pi / 2}$. Note that this decomposition requires qubit 2 being placed in the middle of the linear chain, since it has to perform $\mathrm{CZ}$ gates with both qubit 0 and qubit 1 .

To activate the gate, the magnetic flux $\Phi_{j}(t)$ through the superconducting quantum interference device (SQUID) of coupler $j$ (see Fig. 9) is modulated as

$$
\Phi_{j}(t)=\Theta_{j}+\delta_{j}(t) \cos \left(\omega_{\Phi_{j}} t+\varphi_{j}\right)
$$

where $\Theta_{j}$ is the dc bias, $\delta_{j}(t)$ is an envelope function with sinusoidal rise and fall of $25 \mathrm{~ns}$ and a constant value $\delta_{0_{j}}$ for a time $t_{p}$ in between such that $t_{\text {gate }}=t_{p}+50 \mathrm{~ns}, \omega_{\Phi_{j}}$ is the modulation frequency [close to resonance with the transition frequency between the states that are coupled by the CZ gate (see Fig. 1)], and $\varphi_{j}$ is the initial phase of the drive, which is kept equal to zero until we need to calibrate different values of $\phi$ in the CCZS gate family. Modulating a symmetric SQUID like this results in a time-dependent coupler frequency [103]

$$
\omega_{c_{j}}(t)=\omega_{c_{j}}^{0} \sqrt{\left|\cos \left(\pi \frac{\Phi(t)}{\Phi_{0}}\right)\right|},
$$

where $\Phi_{0}$ is the flux quantum. Our control parameters for the CCZS gate are thus $t_{p}, \delta_{0_{j}}, \Theta_{j}$, and the detuning between $\omega_{\Phi_{j}}$ and the expected resonant frequency.

b. Calibration procedure and results. We calibrate gates in the CCZS family by the following procedure.

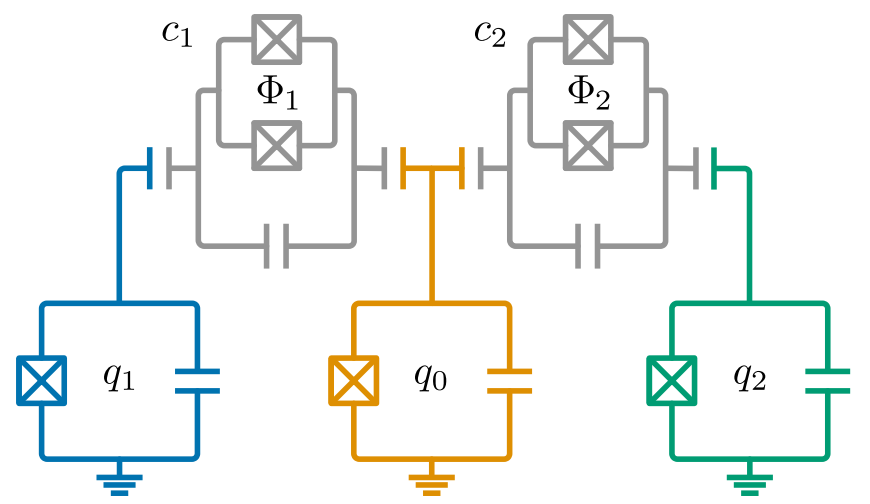

FIG. 9. Setup for implementing the CCZS gate with tunable couplers $c_{1}$ and $c_{2}$ connecting the qubits. The frequency of each coupler $j$ is tuned by changing the magnetic flux $\Phi_{j}$ through the loop formed by the two Josephson junctions.
1. We first tune up high-fidelity $\mathrm{CZ}_{01}$ and $\mathrm{CZ}_{02}$ gates with equal gate times. Both CZ gates must be implemented such that the second excited state used is that of qubit 0, as shown in Fig. 1(b). We begin by exploring the parameter space spanned by $\Theta_{j}$ and $\delta_{0_{j}}$ to find values that yield high population transfers from $\left|1_{0} 1_{j}\right\rangle$ to $\left|2_{0} 0_{j}\right\rangle$. We then plot the population in $\left|22_{0} 0_{j}\right\rangle$ as a function of $t_{\text {gate }}$ and $\omega_{\Phi_{j}}$ as in Figs. 10(a) and 10(c) and go along the value of $\omega_{\Phi_{j}}$ that corresponds to the tip of the resulting chevron pattern to the first value of $t_{\text {gate }}$ that returns all population to $\left|1_{0} 1_{j}\right\rangle$. Finally, we confirm that the $\mathrm{CZ}$ gate fidelity around this point in parameter space is close to $100 \%$ and pick the parameter values in this area that give the highest gate fidelity.

2. Next, we apply pulses to both couplers with the same dc biases and amplitudes as for the good CZ gates found in the previous step, but sweep the modulation frequency of both pulses around the values for the $\mathrm{CZ}$ gates. The smoking gun for the CCZS gates is a maximal population transfer between $|101\rangle$ and $|110\rangle$, which corresponds to $\theta=\pi / 2$. We expect such a point in parameter space to show up at gate times around $\sqrt{2}$ shorter than those found for the $\mathrm{CZ}$ gates. Having found such a point, we check that the gate fidelity around that point is close to $100 \%$ for the $\operatorname{CCZS}(\pi / 2, \phi, 0)$ gate. We then pick the parameter values in this area that give the highest gate fidelity for the desired value of $\phi$.

3. Other elements of the CCZS gate family can also be found, but none of them have such a clear signature as the $|101\rangle \leftrightarrow|110\rangle$ population transfer. In particular, other values for the phase $\phi$ are found by

TABLE I. Parameter values used in Eq. (49) for the simulations of implementing CCZS gates with tunable couplers. Here, $\omega_{c_{j}}^{0}$ is the maximum frequency of coupler $j$. All values are given in units of gigahertz.

\begin{tabular}{ccccccc}
\hline \hline$i$ & $\omega_{i} / 2 \pi$ & $\alpha_{i} / 2 \pi$ & $\omega_{c_{j}}^{0} / 2 \pi$ & $\alpha_{c_{j}} / 2 \pi$ & $g_{i 1} / 2 \pi$ & $g_{i 2} / 2 \pi$ \\
\hline 0 & 4.8 & -0.17 & & & 0.07 & 0.07 \\
1 & 4.225 & -0.18 & 7.8 & -0.12 & 0.07 & \\
2 & 4.35 & -0.18 & 8.0 & -0.12 & & 0.07 \\
\hline \hline
\end{tabular}


(a) Population transfer

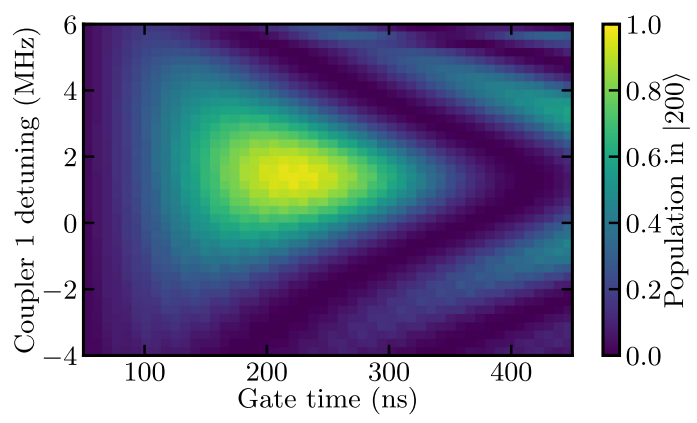

(c)

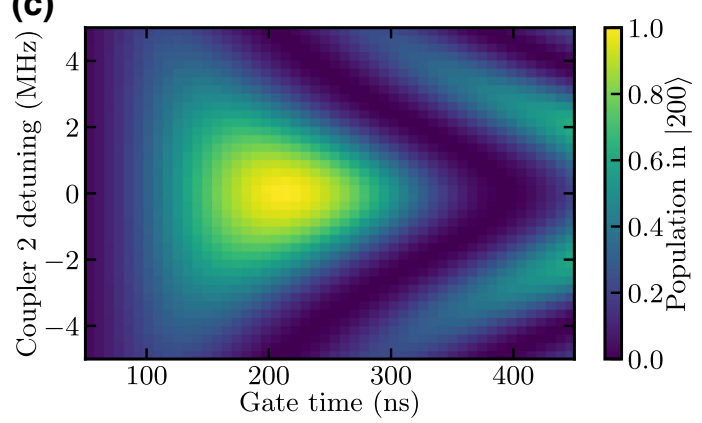

(e)

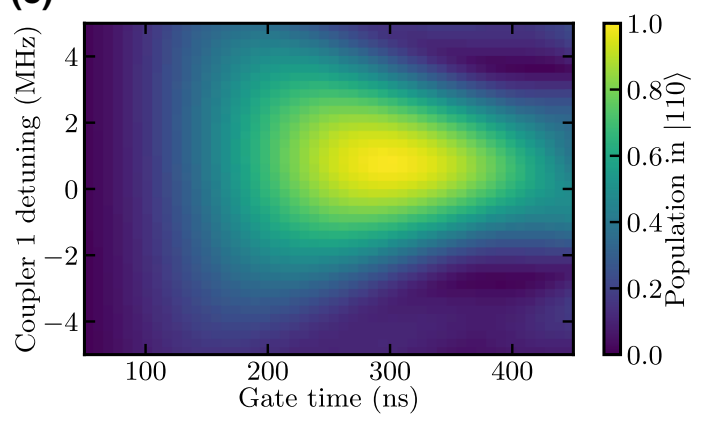

(b) Gate fidelity

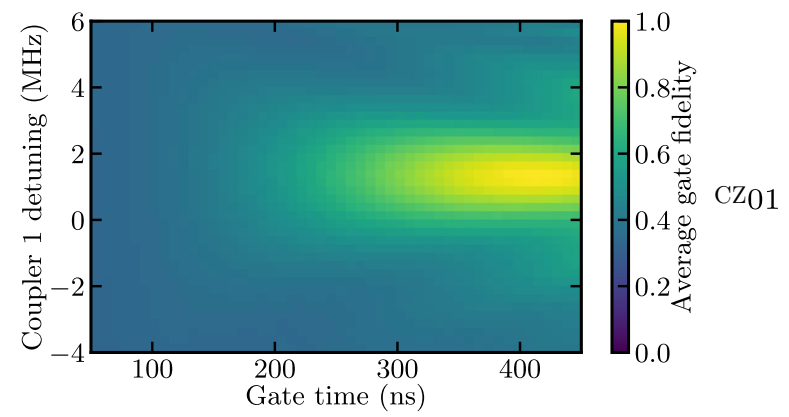

(d)

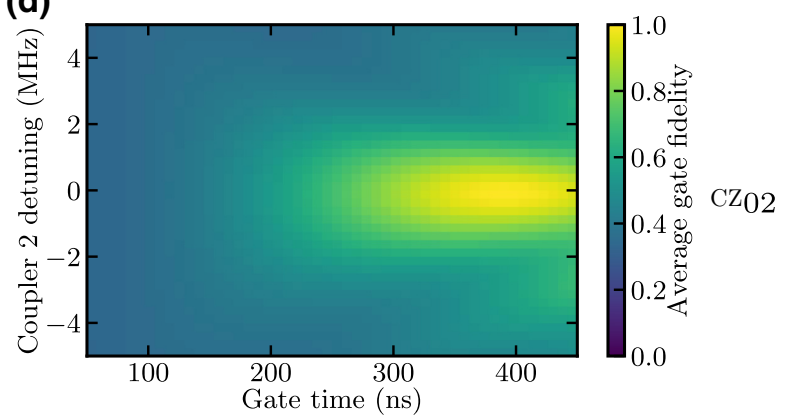

(f)

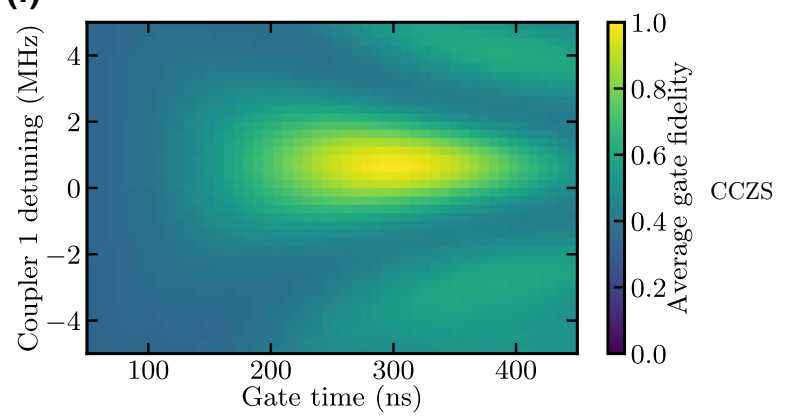

FIG. 10. Calibrating the CCZS gate with tunable couplers. (a) Population in $|200\rangle$ as a function of gate time and the detuning between the modulation frequency $\omega_{\Phi_{1}}$ and the frequency of the transition $|110\rangle \leftrightarrow|200\rangle$ when calibrating the $\mathrm{CZ}_{01}$ gate by initializing the system in $|110\rangle$. (b) The gate fidelity for the $\mathrm{CZ}_{01}$ gate for the parameters in (a). (c) Population in $|200\rangle$ as a function of gate time and the detuning between the modulation frequency $\omega_{\Phi_{2}}$ and the frequency of the transition $|101\rangle \leftrightarrow|200\rangle$ when calibrating the $\mathrm{CZ} Z_{02}$ gate by initializing the system in $|101\rangle$. (d) The gate fidelity for the $\mathrm{CZ}_{02}$ gate for the parameters in (c). (e) Population in $|110\rangle$ as a function of gate time and the detuning between the modulation frequency $\omega_{\Phi_{1}}$ and the frequency of the transition $|110\rangle \leftrightarrow|200\rangle$ when calibrating the $\operatorname{CCZS}(\pi / 2, \pi, 0)$ gate by initializing the system in $|101\rangle$. During the calibration, we also vary $\omega_{\Phi_{2}}$, but in this plot it is kept fixed. (f) The gate fidelity for the $\operatorname{cczS}(\pi / 2, \pi, 0)$ gate for the parameters in (e).

changing the relative initial phase between the two pulses, $\varphi_{1}-\varphi_{2}$. Other values of $\theta$ can, in principle, be found by combining the controls of individual $\mathrm{CZ}$ gates with different gate strengths and adjusting the gate time accordingly [see the discussion below Eq. (26)].

We now show how such a calibration procedure can look like in practice. We first plot the population in $\left|2_{0} 0_{j}\right\rangle$ as a function of $t_{\text {gate }}$ and $\omega_{\Phi_{j}}$ for the two individual $\mathrm{CZ}$ gates in Figs. 10(a) and 10(c). We find high-fidelity (>99.7\% and $>99.9 \%)$ CZ gates with similar gate times, around 405 and $396 \mathrm{~ns}$, by choosing a dc bias $\Theta_{j}=0.275 \Phi_{0}$ for both couplers and an amplitude $\delta_{0_{j}} \approx 0.08 \Phi_{0}$. In Figs. 10(b) and $10(\mathrm{~d})$, we show the corresponding maps of gate fidelity as a function of gate time and $\omega_{\Phi_{j}}$.

We then try applying the same pulses simultaneously. The parameters we vary are now the two modulation frequencies (and the gate time), so we need to look at different projections of the resulting two-dimensional parameter space. In Fig. 10(e), we show one such projection, fixing $\omega_{\Phi_{2}}$ and plotting the population in $|101\rangle$ as a function of $\omega_{\Phi_{1}}$ and $t_{\text {gate. The corresponding gate }}$ fidelity for $\operatorname{CCZS}(\pi / 2, \pi, 0)$ as a function of the same 
parameters is plotted in Fig. 10(f). Selecting the parameters that yield the highest population transfer, and optimizing for fidelity around those values, we find a $\operatorname{cczS}(\pi / 2, \pi, 0)$ gate with $t_{\text {gate }}=291 \mathrm{~ns}$, which is approximately a factor $\sqrt{2}$ shorter plateau time $t_{p}$ than the individual CZ gates. The gate fidelity is $>99.1 \%$.

In the simulations for Fig. 10, we used three energy levels per qubit and tunable coupler to avoid making the computations too heavy. However, all fidelity values quoted for the calibrated gates in the preceding paragraphs are obtained using four energy levels per qubit, ensuring that effects of leakage to higher levels and dispersive shifts resulting from the presence of higher levels are not omitted. Compared to the fidelity values obtained in simulations with three levels per qubit, the values for four levels are only about $0.1 \%$ lower. This slight decrease is not necessarily due to increased leakage or $Z Z$ coupling from the fourth levels, but may largely be attributed to us not having found the optimal operating point for the gate when the numerical simulations took longer to run.

Note that, just as for the simulations with tunable qubits above, we have not included any effects of decoherence in these simulations. The impact of decoherence will be less the faster the gates are. It is possible to calibrate faster $\mathrm{CZ}$ and CCZS gates than the examples shown here in Fig. 10 by choosing other values of $\Theta_{j}$ and $\delta_{0_{j}}$, but we have chosen to show these examples since they illustrate the calibration and workings of the gates more clearly than some of the faster gates.

c. Tuning the gate parameter $\phi$. To further demonstrate the extensive control of gate parameters afforded by the setup with tunable couplers, we calibrate the $\operatorname{CCZS}(\pi / 2, \phi, 0)$ gate for many values of $\phi$ in the range $[0,2 \pi]$. We do this by starting from the optimized parameters for $\operatorname{CCZS}(\pi / 2, \pi, 0)$ found above and then tuning the phase difference $\varphi_{1}-\varphi_{2}$ between the signals modulating the two tunable couplers. The resulting gate fidelities for $\operatorname{CCZS}(\pi / 2, \phi, 0)$ are shown in Fig. 11. For all values of $\phi$ we try, we find gate fidelities above $99 \%$. These high gate fidelities are achieved along the line $\phi=\pi+\varphi_{1}-\varphi_{2}$ $\bmod 2 \pi$, as expected from Eq. (21).

d. Error sources Just as for the setup with tunable qubits in Sec. II E 2, we do not reach perfect $100 \%$ gate fidelity in our simulations of the setup with tunable couplers, despite neglecting decoherence effects. The remaining error has multiple contributions. Firstly, the pulse shape $\delta_{j}(t)$ is chosen to be very simple; no optimal control is applied to improve it. Secondly, higher-order interactions between the qubits mediated by the off-resonant couplers result in $Z Z$ interactions that disturb the three-qubit gate. We observe higher gate fidelities if we allow ourselves to correct phases like those produced by such interactions. This suggests that schemes for reducing unwanted $Z Z$

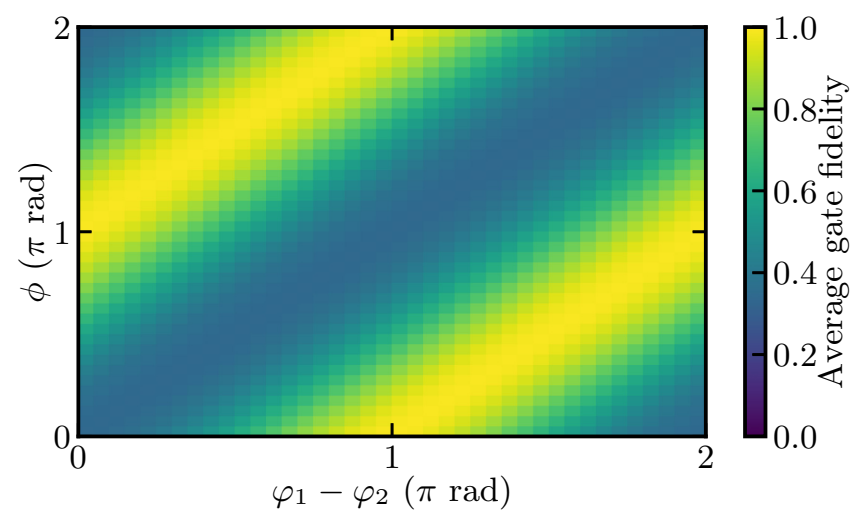

FIG. 11. Calibrating the $\operatorname{CCZS}(\pi / 2, \phi, 0)$ gate by tuning the phase difference $\varphi_{1}-\varphi_{2}$ between the signals modulating the two tunable couplers. The simulations are performed using three energy levels per qubit. The plots shows the gate fidelity for the $\operatorname{CCZS}(\pi / 2, \phi, 0)$ gate as a function of $\phi$ and $\varphi_{1}-\varphi_{2}$. All other parameters are the same as those that gave the highest gate fidelity for $\operatorname{CCZS}(\pi / 2, \pi, 0)$ in Fig. 10. For each value of $\phi$ shown in the plot, the highest gate fidelity exceeds $99 \%$.

interactions in two-qubit gates (see, e.g., Refs. [92,93]) could also be helpful for the three-qubit gate considered here.

Thirdly, we note that we restricted ourselves to calibrating gates of the form $\operatorname{CCZS}(\pi / 2, \phi, 0)$. It is possible that some of the gates we produced had higher gate fidelities with $\operatorname{CCZS}(\theta, \phi, \gamma)$ for other values of $\theta$ and $\gamma$, but we preferred tuning up and showing fidelities for a gate with clearer functionality rather than searching the space of parameters $\theta$ and $\gamma$ to find the highest possible fidelity. Finally, the simulations with five three-level transmon qubits are quite computationally heavy and we needed to search a ten-dimensional parameter space (plateau times $t_{p}$, modulation frequencies $\omega_{\Phi_{j}}$, modulation phases $\phi_{j}$, modulation amplitudes $\delta_{0_{j}}$, and dc biases $\Theta_{j}$ ). There is thus certainly room for improvement in exploration of this parameter space.

\section{SIMULTANEOUS ISWAP GATES}

In this section, we show that the idea of applying simultaneous two-qubit gates to create multiqubit gates is not limited to the CZ gates studied in Sec. II. Here, we investigate what happens when the simultaneous gates are isWAP gates instead. The treatment in this section will be more condensed than in the previous one, since some parts turn out to be quite similar. We note that several other combinations of two-qubit gates are possible, but the detailed study of such possibilities is left for future work.

\section{A. Setup and gate operation}

We consider simultaneous application of isWAP gates that are based on activating a coupling between the states 


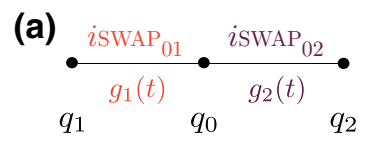

(c)
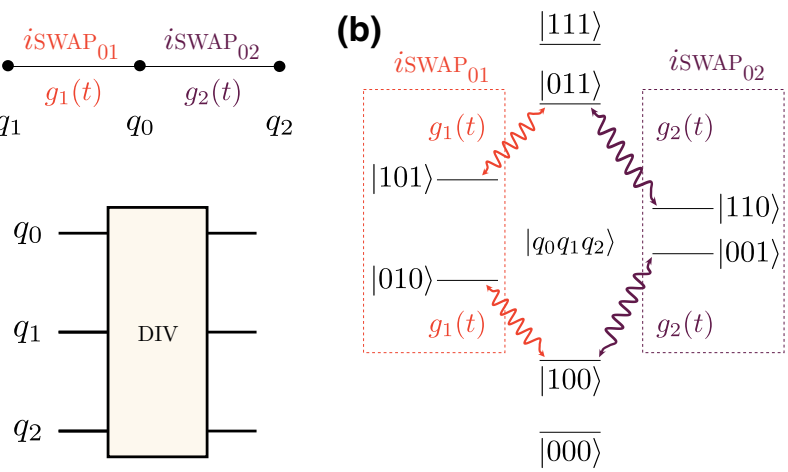

FIG. 12. Setup and operation for the three-qubit gate realized through simultaneous application of two isWAP gates. (a) The setup considered is a linear chain of three qubits with nearestneighbor coupling. The isWAP gates isWAP ${ }_{0 j}$ between qubits 0 and $j=\{1,2\}$ are applied simultaneously by activating couplings between the $\left|1_{0} 0_{j}\right\rangle$ and $\left|0_{0} 1_{j}\right\rangle$ states with coupling strength $g_{j}$. (b) The transitions in the three-qubit system activated by the application of the isWAP gates. The states $|000\rangle$ and $|111\rangle$ are not affected by the gates. (c) We denote the three-qubit operation resulting from the simultaneous application of the two isWAP gates by DIV, since it is a "divider" gate that can distribute one or two excitations among all three qubits.

$|01\rangle$ and $|10\rangle$. In such gates, the states $|00\rangle$ and $|11\rangle$ do not couple to other states and remain unchanged while the states $|01\rangle$ and $|10\rangle$ are swapped and acquire a phase factor $-i$. In superconducting circuits, just as for the $\mathrm{CZ}$ gate, the required coupling can be achieved either by tuning the frequencies of the two qubits into resonance [88,89], possibly in conjunction with tuning a coupler $[92,93]$, by parametrically modulating a tunable coupler between two fixed-frequency qubits $[95,98,107]$, or by parametrically modulating one of the qubits [108,148]. In Ref. [92], a gate fidelity of $99.86 \%$ was reported for a gate time of $30 \mathrm{~ns}$.

\section{Hamiltonians and time evolution}

We consider the same linear chain of three qubits as for the CCZS gate in Fig. 1, but now coupling the transitions between the states $\left|1_{0} 0_{j}\right\rangle$ and $\left|0_{0} 1_{j}\right\rangle$ with strengths $g_{j}$, as illustrated in Fig. 12(a). A simplification compared to the simultaneous CZ gates is that no second excited state of any qubit becomes part of the dynamics. For the simultaneous isWAP gates, only the transitions shown in Fig. 12(b) are activated.

Assuming for simplicity that the transitions shown in Fig. 12(b) are resonant $(\delta=0)$, and that all other transitions are far off resonance, the Hamiltonian for the system can in the interaction picture be written as

$$
H=\sigma_{0}^{10}\left(g_{1} \sigma_{1}^{01}+g_{2} \sigma_{2}^{01}\right)+\text { H.c., }
$$

with operators defined as in Sec. II D 2 a. This Hamiltonian conserves the number of excitations in the system and we also see that the transitions in Fig. 12(b) occur within the subspaces determined by the excitation number.

In the subspaces with one and two excitations, $\Lambda$ or $V$ systems are formed. Just as in Sec. II A 1, it is thus convenient to introduce new basis states that include dark and bright states. Defining

$$
\begin{aligned}
& \left|B_{1}\right\rangle=\frac{g_{1}|10\rangle+g_{2}|01\rangle}{\Omega}, \\
& \left|B_{2}\right\rangle=\frac{g_{2}|10\rangle+g_{1}|01\rangle}{\Omega}, \\
& \left|D_{1}\right\rangle=\frac{g_{2}|10\rangle-g_{1}|01\rangle}{\Omega}, \\
& \left|D_{2}\right\rangle=\frac{g_{1}|10\rangle-g_{2}|01\rangle}{\Omega},
\end{aligned}
$$

where

$$
\Omega=\sqrt{g_{1}^{2}+g_{2}^{2}},
$$

the unitary dynamics generated by the system Hamiltonian in Eq. (52) can be expressed as

$$
\begin{aligned}
U= & |000\rangle\langle 000|+| 111\rangle\langle 111| \\
& +\left|0 D_{2}\right\rangle\left\langle 0 D_{2}|+| 1 D_{1}\right\rangle\left\langle 1 D_{1}\right| \\
& +\cos (\Omega t) I^{\prime}-i \sin (\Omega t) \sigma_{x}^{\prime} \\
& +\cos (\Omega t) I^{\prime \prime}-i \sin (\Omega t) \sigma_{x}^{\prime \prime},
\end{aligned}
$$

where $I^{\prime}$ and $\sigma_{x}^{\prime}$ are defined in the subspace spanned by $|100\rangle$ and $\left|0 B_{1}\right\rangle$, and $I^{\prime \prime}$ and $\sigma_{x}^{\prime \prime}$ are defined in the subspace spanned by $|011\rangle$ and $\left|1 B_{2}\right\rangle$.

\section{The family of three-qubit gates}

The results above show that the states in the computational subspace of the three qubits are affected as follows: $|000\rangle$ and $|111\rangle$ are unchanged, while states are swapped around in the single- and double-excitation subspaces. By introducing the notation $\tan \theta=g_{2} / g_{1}$ (we assume for simplicity that $g_{1}$ and $g_{2}$ are in phase) and $\varphi=\Omega t$, we can write the three-qubit gate, which we denote DIV [see Fig. 12(c)], as

$$
U_{\operatorname{DIV}}(\theta, \varphi)=U_{0} \oplus U_{1}(\theta, \varphi) \oplus U_{2}(\theta, \varphi) \oplus U_{3},
$$

where $U_{j}$ acts on the $j$-excitation subspace. Here, $U_{0}=$ $U_{3}=1$, while $U_{1}$ and $U_{2}$ are found by transforming from the basis with bright and dark states used in Eq. (58) (see Appendix A) to the computational basis; in the singleexcitation subspace spanned by $|010\rangle,|100\rangle$, and $|001\rangle$, we obtain 


$$
U_{1}(\theta, \varphi)=\left[\begin{array}{ccc}
\sin ^{2} \theta+\cos ^{2} \theta \cos \varphi & -i \cos \theta \sin \varphi & \frac{1}{2} \sin 2 \theta(\cos \varphi-1) \\
-i \cos \theta \sin \varphi & \cos \varphi & -i \sin \theta \sin \varphi \\
\frac{1}{2} \sin 2 \theta(\cos \varphi-1) & -i \sin \theta \sin \varphi & \cos ^{2} \theta+\sin ^{2} \theta \cos \varphi
\end{array}\right]
$$

and $U_{2}(\theta, \varphi)$ for the double-excitation subspace spanned by $|101\rangle,|011\rangle$, and $|110\rangle$ has exactly the same form.

An important difference between the DIV gate from simultaneous isWAP gates and the CCZS gate from simultaneous $\mathrm{CZ}$ gates in the previous section is that the operation of the DIV gate never makes any population leave the computational subspace. This is why we can vary the parameter $\varphi$ freely by choosing the evolution time $t$. In the CCZS gate, the evolution time is heavily constrained by the need to ensure that the temporary population in the middle qubit's second excited state returns to the computational subspace at the end of the gate.

\section{Examples of three-qubit gates}

We now study some simple parameter choices for the DIV gate. If we set $g_{1}=g, g_{2}=0$, and $\varphi=\pi / 2$, i.e., $t_{\text {gate }}=\pi / 2 g$, we recover the two-qubit iswAP gate acting on qubits 0 and 1 . In the same way, if $g_{1}=0, g_{2}=g$, and $\varphi=\pi / 2$, we have the two-qubit iswaP gate acting on qubits 0 and 2 .

If we activate both these isWAP interactions simultaneously, i.e., $g_{1}=g_{2}=g$ such that $\theta=\pi / 4$, and choose the gate time $t_{\text {gate }}=\pi / 2 \sqrt{2} g$ such that $\varphi=\pi / 2$, we obtain $\operatorname{DIV}(\pi / 4, \pi / 2)$, for which

$$
U_{1,2}(\pi / 4, \pi / 2)=\left[\begin{array}{ccc}
\frac{1}{2} & -i / \sqrt{2} & -\frac{1}{2} \\
-i / \sqrt{2} & 0 & -i / \sqrt{2} \\
-\frac{1}{2} & -i / \sqrt{2} & \frac{1}{2}
\end{array}\right] .
$$

This gate, which is a factor $\sqrt{2}$ faster than the individual two-qubit isWAP gates, thus takes a single excitation in the middle qubit and divides it evenly between the two outer qubits. A single excitation in one of the outer qubits ends up divided across all three qubits: half in the middle qubit and a quarter in each of the outer qubits.

If we keep the two coupling strengths the same $\left(g_{1}=\right.$ $g_{2}=g$ such that $\left.\theta=\pi / 4\right)$, but vary the parameter $\varphi$ by varying the gate time $t=\varphi / \sqrt{2} g$, the gate becomes

$$
\begin{aligned}
& U_{1,2}(\pi / 4, \varphi) \\
& \quad=\left[\begin{array}{ccc}
\frac{1}{2}(1+\cos \varphi) & -(i / \sqrt{2}) \sin \varphi & \frac{1}{2}(\cos \varphi-1) \\
-(i / \sqrt{2}) \sin \varphi & \cos \varphi & -(i / \sqrt{2}) \sin \varphi \\
\frac{1}{2}(\cos \varphi-1) & -(i / \sqrt{2}) \sin \varphi & \frac{1}{2}(1+\cos \varphi)
\end{array}\right] .
\end{aligned}
$$

If we instead fix $\varphi=\pi / 2$, but vary $\theta$ by varying the ratio of the coupling strengths $g_{1}$ and $g_{2}$, the resulting gate is given by

$$
U_{1,2}(\theta, \pi / 2)=\left[\begin{array}{ccc}
\sin ^{2} \theta & -i \cos \theta & -\frac{1}{2} \sin 2 \theta \\
-i \cos \theta & 0 & -i \sin \theta \\
-\frac{1}{2} \sin 2 \theta & -i \sin \theta & \cos ^{2} \theta
\end{array}\right] .
$$

\section{B. Decomposition into two-qubit gates}

We note that all the three-qubit gates in Sec. III A 3 entangle all three qubits. Although finding a decomposition of the DIV gate into single- and two-qubit gates is less straightforward than for the CCZS gate in Sec. II B, we can from this entanglement conclude that at the very least two sequential two-qubit gates are necessary for such a decomposition. Since the three-qubit gate is already faster than a single two-qubit gate, this guarantees a significant speedup.

\section{Constructing other three-qubit gates}

Unlike the CCZS gate, the DIV gate cannot be interpreted as one qubit controlling a two-qubit operation on the other two qubits. The most well-known three-qubit gates, the Fredkin, iFredkin, and Toffoli gates considered in Sec. III $\mathrm{C}$, are all such gates. It is thus clear that the DIV gate cannot be equivalent to any of these three-qubit gates for any choice of parameters $\theta$ and $\varphi$. Furthermore, it does not appear possible to change any DIV gate into such a form by adding a single two-qubit gate before or after the DIV gate.

\section{Creating large entangled states}

We now turn to how the DIV gate and its generalizations to more qubits can be used to rapidly create large entangled states, similar to what we showed for the CCZS gate in Sec. II D. We first note that arbitrary superpositions of all permutations of three-qubit states with one excitation can easily be created by starting from $|000\rangle$, exciting qubit 0 , and then applying the DIV gate for suitable values of $\theta$ and $\varphi$, yielding

$$
\begin{aligned}
& U_{1}(\theta, \varphi)|100\rangle \\
& \quad=\cos \varphi|100\rangle-i \sin \varphi(\cos \theta|010\rangle+\sin \theta|001\rangle) .
\end{aligned}
$$

In particular, the three-qubit $\mathrm{W}$ state

$$
\left|D_{3}^{1}\right\rangle=\frac{1}{\sqrt{3}}(|100\rangle+|010\rangle+|001\rangle)
$$




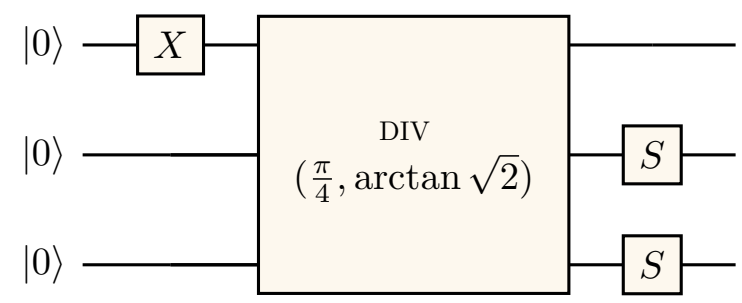

FIG. 13. Quantum circuit for generating the three-qubit $W$ state $(|100\rangle+|010\rangle+|001\rangle) / \sqrt{3}$ using the DIV gate. The phase gate $S$ is $\sqrt{Z}$.

can be constructed by choosing $\theta=\pi / 4$ (i.e., $g_{1}=g_{2}$ ) and $\varphi=\arctan \sqrt{2}$ in Eq. (64), and following that by applying single-qubit gates to qubits 1 and 2, as shown in Fig. 13 .

We note that the experiment in Ref. [121] showed how to construct a $\mathrm{W}$ state by single-qubit rotations and a single application of simultaneous isWAP interactions between all three qubits in a triangular setup instead of the linear chain considered here. A generalization of this protocol to more qubits is given in Ref. [155]. Furthermore, a protocol to construct the three-qubit GHZ state using single-qubit rotations and a single application of simultaneous isWAP interactions in a linear chain like we consider here was presented in Ref. [156].

The multiqubit version of the simultaneous isWAP interaction, where the dynamics of a central qubit 0 and its $N$ nearest neighbors $\{j\}$ are governed by the interactionpicture Hamiltonian

$$
H=\sigma_{0}^{10} \sum_{j=1}^{N} g_{j} \sigma_{j}^{01}+\text { H.c. }
$$

is the same as the simultaneous $\mathrm{CZ}$ interaction given in Eq. (39) except that it is the $|0\rangle \leftrightarrow|1\rangle$ transition that couples to the surrounding qubits instead of the $|1\rangle \leftrightarrow|2\rangle$ transition. We can thus reuse much of what we derived in Sec. II D 2 about how to rapidly create large Dicke states. For example, the procedure for creating W states with many qubits described in Fig. 6 can also be implemented with simultaneous isWAP gates. It is actually even easier, since the initial qubit only needs to be prepared in state $|1\rangle$ instead of $|2\rangle$ and the later step of flipping $|1\rangle$ to $|2\rangle$ for other qubits described there can be omitted.

To create superpositions of Dicke states like in Sec. II D 2, only a minor modification of the protocol presented there is needed to adapt it to simultaneous isWAP gates. We simply change the states $\left|0_{0}\right\rangle,\left|1_{0}\right\rangle$, and $\left|2_{0}\right\rangle$ to $\left|2_{0}\right\rangle,\left|0_{0}\right\rangle$, and $\left|1_{0}\right\rangle$, respectively, during the execution of the protocol. At the end, we change them back to obtain the state in Eq. (43).

\section{E. Experimental feasibility}

In the same way as for the CCZS gate (see Sec. II E), there are several experimental setups with superconducting qubits that could be used to implement the simultaneous isWAP gates that make up the DIV gate. This includes setups with tunable qubits, where the states $|01\rangle$ and $|10\rangle$ are tuned into resonance [88,89]. This activation of the isWAP gate can be further enhanced with a tunable coupler $[92,93]$. The other type of setup uses parametric modulation of either a tunable coupler $[95,98,107]$ or one of the qubits $[108,148]$.

For brevity and simplicity, we here limit our simulations to the implementation with tunable qubits. In this implementation, the parameter $\theta$ is fixed by the coupling strengths set in the hardware and cannot be changed in experiment. An implementation with parametric modulation of tunable couplers instead would enable controlling $\theta$ in situ. Such an implementation can be calibrated in similar fashion as the CCZS gate with tunable couplers in Sec. II E 2.

For the implementation with tunable qubits, we consider the same setup and parameters as in Sec. II E 1 except that we increase the maximum energy of qubit 2 to $\omega_{0}$; see Fig. 7(a) and Eqs. (46)-(48) with $g_{j}=\lambda_{j} / 2$. We further use the same pulse shapes as in Fig. 7(b), but now tuning $\omega_{1}$ and $\omega_{2}$ into resonance with $\omega_{0}$ instead of $\omega_{0}+\alpha_{0}$ and adapting the gate times to yield the isWAP and DIV gates, resulting in the tuning shown in Fig. 14(a).

We first tune up the individual isWAP $\mathrm{P}_{01}$ and isWAP $\mathrm{P}_{02}$ gates by tuning just one of the outer qubits into resonance with the middle qubit. For the isWAP 01 gate, we find a gate fidelity of $99.8 \%$ with a gate time $t_{\text {gate, }, \mathrm{SWAP}_{01}}=66.8 \mathrm{~ns}$, and for the isWAP 02 gate, we find a gate fidelity of $99.6 \%$ using the same gate time.

We then tune up the $\operatorname{DIV}(\pi / 4, \pi / 2)$ gate [see Eq. (61)] by tuning both outer qubits into resonance with the middle qubit in a synchronized fashion, reducing the gate time by around a factor $\sqrt{2}$. Note that other values of $\varphi$ are easily implemented by decreasing or increasing the gate time, while $\theta=\pi / 4$ is fixed by the choice of $g_{1}=g_{2}$ used here. For the $\operatorname{DIV}(\pi / 4, \pi / 2)$ gate, we find a gate fidelity of $99.68 \%$ for the gate time $t_{\text {gate, DIV }}=47.5 \mathrm{~ns} \approx$ $t_{\text {gate, iSWAP }} / \sqrt{2}$. Just as in Sec. II E, the gate fidelity is calculated without including any effects of decoherence in the simulation. To illustrate the calibrated gate, we plot in Fig. 14(b) the population transfers in the single-excitation subspace when the system is initialized in the state $|010\rangle$.

As in Sec. II E 1, we check the impact on gate fidelity of deviations from design values for the coupling strengths. We optimize the $\operatorname{DIV}(\pi / 4, \pi / 2)$ gate with $g_{2}=$ $1.1 g_{1}$ (instead of $g_{2}=g_{1}$ that we used above) and find a gate fidelity of $99.71 \%$. For the DIV $\left(\arctan g_{2} / g_{1}, \pi / 2\right)$ gate, which should be best suited to unequal couplings, we find a fidelity of $99.69 \%$. It thus appears that the small 
(a)

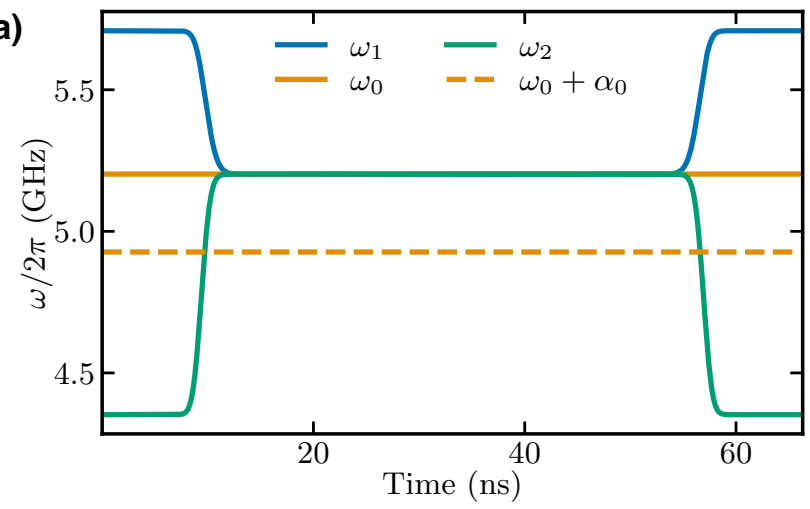

(b)

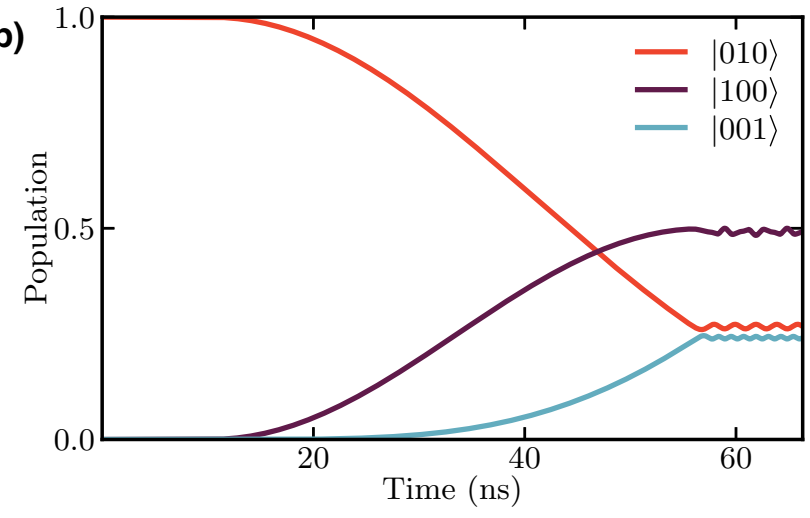

FIG. 14. Implementing the $\operatorname{DIV}(\pi / 4, \pi / 2)$ gate with tunable qubits. (a) The tuning of the qubit energies used to realize the gate. (b) Population of the states $|100\rangle,|010\rangle$, and $|001\rangle$ (the single-excitation subspace) as a function of time during the gate when the initial state is $|010\rangle$. As Eq. (61) shows, the effect of the gate is to divide an excitation in one of the outer qubits such that half ends up in the middle qubit and a quarter each in the outer qubits.

variation in coupling strength, which corresponds to a change in angle $\theta$ for the gate of just $2.7^{\circ}$, does not significantly impact the fidelity, which rather seems to depend on the optimization of the tuning of the qubits in and out of the resonance of the gate.

The deviation from perfect gate fidelity for the gate in Fig. 14 can be attributed to several factors, similar to the case of the CCZS gate with tunable qubits in Sec. II E 1. As we tune qubit 2 into resonance with qubit 0 , we pass the point where $\omega_{2}=\omega_{0}+\alpha_{0}$, i.e., where the $\left|1_{0} 1_{2}\right\rangle \leftrightarrow$ $\left|2_{0} 0_{2}\right\rangle$ transition is resonant. Similarly, when qubit 1 is tuned into resonance with qubit 0 , it passes the point where $\omega_{1}+\alpha_{1}=\omega_{0}$, i.e., where the $\left|1_{0} 1_{1}\right\rangle \leftrightarrow\left|0_{0} 2_{1}\right\rangle$ transition is resonant. These transitions can cause leakage from the computational subspace. Data from simulations with the initial state being $|110\rangle,|101\rangle$, and $|011\rangle$, shown in Appendix B, support this explanation. When the initial state is $|110\rangle(|101\rangle)$, we observe an increase of the $|020\rangle$ $(|200\rangle)$ population of about $1 \times 10^{-2}\left(5 \times 10^{-3}\right)$ occurring at $t \approx 10 \mathrm{~ns}$, consistent with a brief activation of the $\left|1_{0} 1_{1}\right\rangle \leftrightarrow\left|0_{0} 2_{1}\right\rangle\left(\left|1_{0} 1_{2}\right\rangle \leftrightarrow\left|2_{0} 0_{2}\right\rangle\right)$ transition. When the initial state is $|011\rangle$, any population transfers during the initial tuning into resonance are instead at least one order of magnitude lower. Just like for the CCZS gate, it would likely be beneficial to apply more advanced methods for optimizing the tuning of the qubits in and out of resonance.

\section{CONCLUSION}

We have shown how multiqubit gates can be constructed and implemented by simultaneously applying two-qubit gates to a group of qubits such that at least one qubit is affected by the operation of two or more of these two-qubit gates. The resulting multiqubit gates are as fast as, and in many cases clearly faster than, the individual two-qubit gates on their own. Furthermore, the multiqubit gates can have larger entangling power than the sequential application of the constituent two-qubit gates, in addition to being much faster than such a sequential application.

Since our scheme for multiqubit gates only relies on control operations corresponding to two-qubit gates, our ideas are ready to be implemented in existing quantum hardware without the need for any additional components, complicated pulse shapes, hardware redesign, or other changes beyond some recalibration of the lengths (and in some cases, the phases) of the control pulses already optimized for two-qubit gates. This means that the multiqubit gates presented in this article, and other multiqubit gates using the same principles, could become useful immediately across mature quantum-computing platforms like superconducting circuits, trapped ions, and others.

We illustrated our ideas for multiqubit gates with two specific examples: simultaneously applied interactions for CZ gates and simultaneously applied interactions for isWAP gates. For the simultaneous $\mathrm{CZ}$ gates, based on activating the $|11\rangle \leftrightarrow|02\rangle$ transition, we showed that applying them to the nearest neighbors in a linear chain of three qubits, with the middle qubit being the one excited to $|2\rangle$, resulted in a three-qubit gate that we denoted CCZS. This CCZS gate applies a combination of the $\mathrm{CZ}$ and SWAP gates to the outer qubits in the chain conditioned on the middle qubit being in its excited state. By controlling the ratio of amplitudes and the relative phases of the pulses for the constituent $\mathrm{CZ}$ gates, and also the detuning from resonance of the $|11\rangle \leftrightarrow$ |02) transition, we gain access to a whole family of CCZS gates. For the case where the $\mathrm{CZ}$ control pulses are in phase, on resonance, and of equal amplitude, the gate time for the CCZS gate is a factor $\sqrt{2}$ shorter than for a single $\mathrm{CZ}$ gate.

Exploring the entangling power of the CCZS gates, we showed that a decomposition of a gate in the CCZS family in general requires three sequential two-qubit gates. We also demonstrated that gates from the CCZS family can be used to construct other three-qubit gates: the iFredkin and Fredkin gates are equivalent to a CCZS gate followed by a two-qubit $\mathrm{CZ}$ gate or a three-qubit CCZ gate, respectively. For the iFredkin gate, this suggests that we can implement 
it twice as fast using a construction with a CCZS gate than a standard decomposition into two-qubit gates. Furthermore, we showed that a single CCZS gate combined with a few single-qubit gates can be used to construct an entangled three-qubit GHZ state. Finally, we generalized the CCZS gate operation to more qubits and showed that, in combination with a few single-qubit gates, it can create large entangled Dicke states in very few steps.

For the simultaneous isWAP gates, based on activating the $|01\rangle \leftrightarrow|10\rangle$ transition, we showed that, when they are applied in a linear chain of three qubits, a three-qubit gate that we denoted DIV is created. The DIV gate distributes excitations among the three qubits within the one- and two-excitation subspaces while leaving the states $|000\rangle$ and |111) unchanged. We showed that we can create a large family of DIV gates by controlling the gate time and the relative strength of the two constituent iswaP gates. Furthermore, similar to the CCZS gates, we showed that the DIV gates are in general faster than single isWAP gates and can be used to rapidly construct large entangled states like Dicke and GHZ states.

For both the CCZS gate and the DIV gate, we performed numerical simulations using parameters from existing state-of-the-art quantum hardware with superconducting qubits to demonstrate that these three-qubit gates are ready to be implemented with high fidelity in experiments. For the CCZS gate, we showed that it can be implemented with both tunable qubits and with tunable couplers, where the latter gives some more freedom to control parameters and realize the whole family of gates. We found that both setups enable gate fidelities exceeding $99.3 \%$, with the tunable qubits reaching a gate fidelity above $99.4 \%$. For the DIV gate, we limited the simulations to the setup with tunable qubits and demonstrated a gate fidelity exceeding $99.6 \%$. We emphasize that all these simulations used quite simple and straightforward methods for optimization and calibration, indicating that these high gate fidelities should be within reach in experiment. Furthermore, we identified factors contributing to the deviations from perfect gate fidelity, e.g., lack of optimal control applied to gate parameters varying in time and the presence of unwanted $Z Z$ coupling. This allowed us to suggest several improvements to the operation of the three-qubit gates, which should enable even higher gate fidelities than demonstrated here.

In conclusion, we have introduced a general method for creating multiqubit gates using two-qubit gates already in use in current quantum hardware. We have shown that these multiqubit gates are fast, powerful, and ready to be implemented in existing experimental setups without any significant modifications needed. This opens up a wealth of possible applications by making quantum circuits more compact and faster to run, which is crucial for unleashing the potential of NISQ devices that are limited by coherence times.

\section{OUTLOOK}

We see at least five directions for further research building on the results presented in this article. The first is to test the ideas detailed here in actual experiments. Such experiments could be performed using various setups with superconducting qubits, as we have analyzed in Secs. II E and III E, but also on other quantum hardware platforms. We note that experimental implementations would benefit from further developing calibration methods compared to what we showed in Secs. II E and III E. In the numerical simulations there, we had access to the full propagator associated with the gate. This allowed us to simplify the calibration process substantially, since we could easily check the average gate fidelity for those points in parameter space that showed population transfers between the computational states corresponding to the gate we sought.

The question of experiments ties into the second research direction, which is to analyze how well the schemes from this article will perform on other platforms than superconducting qubits, e.g., trapped ions and Rydberg atoms. Furthermore, it should be investigated whether there are two-qubit gate implementations native to these other platforms that can be run simultaneously to create new multiqubit gates.

This last part can also be viewed as part of the third research direction, which is to find more multiqubit gates realized through simultaneous application of other twoqubit gates than $\mathrm{CZ}$ and iSWAP, which were used as examples in this article. Candidates for such two-qubit gates include CNOT gates implemented through cross-resonance driving $[157,158]$. It may also be possible to simultaneously apply different two-qubit gates to different pairs of qubits to create yet other multiqubit gates.

The fourth research direction we envision is to compile or transpile various quantum algorithms anew with the novel multiqubit gates included in the native gate set of the device that the algorithm is to be executed on. We expect this to lead to a significantly reduced circuit depth and run time for some algorithms. As mentioned in the Introduction, the CCZS gate seems particularly suited to improve phase estimation and spectrum qubitization, but there are likely many more algorithms that would benefit from its inclusion. For example, one could investigate the use of our multiqubit gates in the entangling layers of variational quantum algorithms [12] like the quantum approximate optimization algorithm [159], the quantum alternating operator ansatz [86], or the variational quantum eigensolver [160].

Finally, we also believe that tools from optimal control and insights from other works on optimizing pulse shaping for gates should be applied to the multiqubit gates developed here. This could help achieve even higher gate fidelities and shorter circuit run times by reducing leakage 
to states outside the computational subspace and further decreasing the gate time.

\section{ACKNOWLEDGMENTS}

We acknowledge support from the Knut and Alice Wallenberg Foundation through the Wallenberg Centre for Quantum Technology (WACQT) and from the EU Flagship on Quantum Technology H2020-FETFLAG-2018-03 project 820363 OpenSuperQ. The quantum circuits shown were drawn using quantikz [161].

Note added.-We recently became aware of patent application PCT/US2019/016047 from Niu et al. at Google [162], who independently found the idea of the $\operatorname{CCZS}(\theta, \pi, 0)$ gate. In their realization, the only parameter that can be tuned is $\theta$, which is set by the ratio of the amplitudes of the coupling strengths for the two $|11\rangle \leftrightarrow|02\rangle$ transitions involved in the gate.

After this manuscript was submitted for publication, the preprint of Ref. [163] appeared on arXiv. In that preprint, it was experimentally demonstrated that simultaneous crossresonance driving on a chain of three superconducting qubits (as suggested in Sec. V above) yields a three-qubit iToffoli gate.

\section{APPENDIX A: SIMULTANEOUS CZ GATES WITH COUPLING BETWEEN QUBITS 1 AND 2}

In this appendix, we consider the setup from Sec. II A with the addition of a direct coupling between qubits 1 and 2. In superconducting circuits, such a coupling can arise due to a small capacitance connecting the two distant qubits or due to a tailored all-to-all coupling in a triangular lattice [164].

For setups where the $\mathrm{CZ}$ gates are performed by tuning the states $\left|1_{0} 1_{j}\right\rangle$ and $\left|2_{0} 0_{j}\right\rangle$ into resonance, the states $|x 01\rangle$ and $|x 10\rangle$ with $x=\{0,1,2\}$ will also become resonant. Then, a direct coupling between qubits 1 and 2 will activate an isWAP gate between them. If the $\mathrm{CZ}$ gates are instead performed through parametric modulation of tunable couplers between the three qubits, such an iswAP gate will be activated if there is a frequency component of the modulation in the coupler connecting qubits 1 and 2 that matches the energy difference between the states $|x 01\rangle$ and $|x 10\rangle$.

We thus have three additions to the diagram in Fig. 1(b): a coupling between $|101\rangle$ and $|110\rangle$, transforming the effective $\Lambda$ system in the upper part of the figure into a $\Delta$ system; a coupling between $|201\rangle$ and $|210\rangle$, transforming the effective $V$ system in the lower part of the figure into a $\nabla$ system; and a coupling between $|001\rangle$ and $|010\rangle$. The effect of the last part is simply to change the first term in Eq. (19) from $|0\rangle\left\langle\left. 0\right|_{0} \otimes \mathbb{I}_{1} \otimes \mathbb{I}_{2}\right.$ to $\left.\mid 0\right\rangle\left\langle\left. 0\right|_{0} \otimes U_{\text {iSWAP }}(\beta)\right.$, where

$$
U_{\mathrm{iSWAP}}(\beta)=\left[\begin{array}{cccc}
1 & 0 & 0 & 0 \\
0 & \cos \beta & -i \sin \beta & 0 \\
0 & -i \sin \beta & \cos \beta & 0 \\
0 & 0 & 0 & 1
\end{array}\right]
$$

and the angle $\beta=g t$ is determined by the interaction strength $g$ between $|001\rangle$ and $|010\rangle$ and the gate time $t$. In the following, we therefore investigate the dynamics of the effective three-level systems, which will determine the gate time, since we must make sure to return all population to the computational subspace at the end of the gate.

To clarify the dynamics in the full system, we now model the $\Delta$ system separately as in Ref. [165]. For brevity, the states $|101\rangle,|200\rangle$, and $|110\rangle$ are renamed $|1\rangle$, $|2\rangle$, and $|3\rangle$, respectively. Denoting the coupling strength on the transition $|i\rangle \leftrightarrow|j\rangle$ by $\alpha_{i j}$ (note that $g=\alpha_{13}$ ) and assuming that this transition is activated by parametrically modulating the coupling strength with time dependence $\cos \left(v_{i j} t+\phi_{i j}\right)$, the Hamiltonian for the $\Delta$ system can be written as

$$
\begin{aligned}
H_{\Delta}= & 2 \alpha_{12}\left(|1\rangle\langle 2| e^{i \theta_{12}}+\text { H.c. }\right) \cos \left(v_{12} t+\phi_{12}\right) \\
& +2 \alpha_{23}\left(|2\rangle\langle 3| e^{i \theta_{23}}+\text { H.c. }\right) \cos \left(v_{23} t+\phi_{23}\right) \\
& +2 \alpha_{13}\left(|1\rangle\langle 3| e^{i \theta_{13}}+\text { H.c. }\right) \cos \left(v_{13} t+\phi_{13}\right) \\
& +\sum_{i} \omega_{i}|i\rangle\langle i|,
\end{aligned}
$$

where $\omega_{i}$ is the energy of state $|i\rangle$. Transforming to a suitable rotating frame using the transformation

$$
\begin{aligned}
T=\exp [ & i\left(v_{12} t+\phi_{12}-\theta_{12}\right)|1\rangle\langle 1| \\
& \left.+i\left(v_{23} t+\phi_{23}+\theta_{23}\right)|3\rangle\langle 3|\right],
\end{aligned}
$$

making the rotating-wave approximation, assuming that $v_{13}=v_{12}-v_{23}$, and shifting the zero energy to $\omega_{2}$, we obtain

$$
\begin{aligned}
\tilde{H}_{\Delta}= & \alpha_{12}(|1\rangle\langle 2|+| 2\rangle\langle 1|) \\
& +\alpha_{23}(|2\rangle\langle 3|+| 3\rangle\langle 2|) \\
& +\alpha_{13}\left(|1\rangle\left\langle 3\left|e^{i \Phi}+\right| 3\right\rangle\langle 1| e^{-i \Phi}\right) \\
& +\Delta_{1}|1\rangle\left\langle 1\left|+\Delta_{3}\right| 3\right\rangle\langle 3|,
\end{aligned}
$$

where

$$
\begin{aligned}
\Delta_{1} & =\omega_{1}-\omega_{2}-v_{12}, \\
\Delta_{3} & =\omega_{3}-\omega_{2}-v_{23}, \\
\Phi & =\phi_{12}-\theta_{12}-\phi_{23}-\theta_{23}+\theta_{13}-\phi_{13} .
\end{aligned}
$$


Further simplifying the situation by assuming that $\Delta_{1}=$ $\Delta_{3}=0$ (resonant individual CZ gates) and $\Phi=0$, introducing the dark and bright states

$$
\begin{aligned}
& |B\rangle=\sin \theta|1\rangle+\cos \theta|3\rangle, \\
& |D\rangle=\cos \theta|1\rangle-\sin \theta|3\rangle,
\end{aligned}
$$

where $\tan \theta=\alpha_{12} / \alpha_{23}$, and then also assuming that the two individual $\mathrm{CZ}$ gates have equal gate strength $\left(\alpha_{12}=\right.$ $\alpha_{23}$, so $\theta=\pi / 4$ ), we arrive at

$$
\tilde{H}_{\Delta}=\sqrt{2} \alpha_{12}(|B\rangle\langle 2|+| 2\rangle\langle B|)+\alpha_{13}(|B\rangle\langle B|-| D\rangle\langle D|) .
$$

In the space spanned by $|B\rangle,|2\rangle$, and $|D\rangle$, this Hamiltonian can be written in matrix form as

$$
\begin{aligned}
\tilde{H}_{\Delta} & =\left[\begin{array}{ccc}
\alpha_{13} & \sqrt{2} \alpha_{12} & 0 \\
\sqrt{2} \alpha_{12} & 0 & 0 \\
0 & 0 & -\alpha_{13}
\end{array}\right] \\
& =\frac{\alpha_{13}}{2} \mathbb{I}+\left[\begin{array}{ccc}
\alpha_{13} / 2 & \sqrt{2} \alpha_{12} & 0 \\
\sqrt{2} \alpha_{12} & -\alpha_{13} / 2 & 0 \\
0 & 0 & -3 \alpha_{13} / 2
\end{array}\right] .
\end{aligned}
$$

From Eq. (A11), we see that the states $|B\rangle$ and $|2\rangle$ form a two-level system with dynamics governed by the Hamiltonian

$$
H_{2}=\frac{\alpha_{13}}{2} \sigma_{z}+\sqrt{2} \alpha_{12} \sigma_{x}=\Omega \vec{n} \cdot \vec{\sigma}
$$

$$
U_{\text {bare }}=\left[\begin{array}{ccc}
-\frac{1}{2} e^{-i \alpha_{13} t / 2}\left(1-e^{-i \alpha_{13} t / 2}\right) & 0 & -\frac{1}{2} e^{-i \alpha_{13} t / 2}\left(1+e^{-i \alpha_{13} t / 2}\right) \\
0 & -e^{-i \alpha_{13} t / 2} & 0 \\
-\frac{1}{2} e^{-i \alpha_{13} t / 2}\left(1+e^{-i \alpha_{13} t / 2}\right) & 0 & -\frac{1}{2} e^{-i \alpha_{13} t / 2}\left(1-e^{-i \alpha_{13} t / 2}\right)
\end{array}\right]
$$

We see from Eq. (A19) that a full population transfer between $|101\rangle$ and $|110\rangle$ requires either $t=4 \pi / \alpha_{13}$ or $\alpha_{13}=0$. The latter is the case treated in the main text. The former condition shows that a three-qubit gate similar to the CCZS gate in Sec. II can also be implemented when there is an additional nonzero direct coupling $\alpha_{13}$ between qubits 1 and 2 .

We finally note that the analysis here for the $\Delta$ system also applies to the $\nabla$ system, if we identify the states $|1\rangle,|2\rangle$, and $|3\rangle$ with $|210\rangle$, $|111\rangle$, and $|201\rangle$, respectively. This means that the condition $\Omega t=\pi$ imposed above also ensures that no leakage from the computational subspace takes place in the $\nabla$ system, since where

$$
\begin{aligned}
& \Omega=\sqrt{\left(\frac{\alpha_{13}}{2}\right)^{2}+2 \alpha_{12}^{2}}, \\
& \vec{n}=\left(\frac{\sqrt{2} \alpha_{12}}{\Omega}, 0, \frac{\alpha_{13}}{2 \Omega}\right), \\
& \vec{\sigma}=\left(\sigma_{x}, \sigma_{y}, \sigma_{z}\right) .
\end{aligned}
$$

Thus, including the phase, the time evolution of $|B\rangle$ and $|2\rangle$ is given by

$$
e^{-i \alpha_{13} t / 2}[I \cos (\Omega t)-\sin (\Omega t) \vec{n} \cdot \vec{\sigma}],
$$

while $|D\rangle$ acquires a phase $e^{i \alpha_{13} t}$.

Since we want to eliminate leakage to the $|200\rangle=|2\rangle$ state, which is outside of the computational subspace, we need the off-diagonal elements in Eq. (A16) to be zero. This is achieved when $\Omega t=\pi$. Then, the time evolution for $|B\rangle,|2\rangle$, and $|D\rangle$ is given by

$$
U=\left[\begin{array}{ccc}
-e^{-i \alpha_{13} t / 2} & 0 & 0 \\
0 & -e^{-i \alpha_{13} t / 2} & 0 \\
0 & 0 & e^{-i \alpha_{13} t}
\end{array}\right]
$$

Transforming back to the bare basis of $|1\rangle,|2\rangle$, and $|3\rangle$, using the fact that the transformation matrix for going from $|1\rangle,|2\rangle$, and $|3\rangle$ to $|B\rangle,|2\rangle$, and $|D\rangle$ is

$$
T=\left[\begin{array}{ccc}
\sin \theta & 0 & \cos \theta \\
0 & 1 & 0 \\
\cos \theta & 0 & -\sin \theta
\end{array}\right]
$$

we obtain

the only effect on the state $|111\rangle$ is that it acquires a phase factor $-\exp \left(-i \alpha_{13} t / 2\right)$, as shown by Eq. (A19).

\section{APPENDIX B: FURTHER DATA ON POPULATION TRANSFERS IN GATE IMPLEMENTATIONS WITH TUNABLE QUBITS}

In this appendix, we present additional data from our simulations in Secs. II E 1 and III E of the possible implementations of the CCZS and DIV gates with tunable qubits. These data shed light on unwanted population transfers that can occur along the way, and thus decrease gate 
fidelity, when the qubits are tuned into the resonance corresponding to the three-qubit gate. We also discuss the possibility of population transfers to other states than those shown in the data here.

In Fig. 15, we show the populations of the singleexcitation states $|100\rangle,|010\rangle$, and $|001\rangle$ when the $\operatorname{CCZS}(\pi / 2, \pi, 0)$ gate is implemented as shown in Fig. 7 in Sec. III E 1 for these three initial states. From the data in Figs. 15 (a) and 15 (b), we see that roughly $0.4 \%$ of the population in the initial states $|100\rangle$ and $|010\rangle$ is transferred to the other state after about $10 \mathrm{~ns}$, which is when qubit 1 is tuned through the point where $\omega_{1}=\omega_{0}$, i.e., where the states $|100\rangle$ and $|010\rangle$ are on resonance. For the case when the initial state is $|001\rangle$, shown in Fig. 15(c), the amount of population lost is more than an order of magnitude less, since $\omega_{2}$ does not pass through any similar resonance during the initial tuning phase of the three-qubit gate.

We also observe a small population transfer slowly taking place between the states $|010\rangle$ and $|001\rangle$ in Figs. 15(b) and $15(\mathrm{c})$, beginning when the qubits have been tuned into resonance such that $\omega_{1}=\omega_{2}$. We attribute this population transfer to a dispersive coupling between qubits 1 and 2 mediated through qubit 0 , which is coupled to both of them and is detuned from both of them by $\alpha_{0}$. However, we note that this population transfer, although accumulated over the whole gate instead of just during the initial tuning phase, is clearly a smaller effect than the population transfers discussed in the preceding paragraph, as is most clearly seen in Fig. 15(b).

In Fig. 16, we show the populations of the doubleexcitation states $|110\rangle,|101\rangle,|011\rangle,|200\rangle,|020\rangle$, and $|002\rangle$ when the $\operatorname{DIV}(\pi / 4, \pi / 2)$ gate is implemented as shown in Fig. 14 in Sec. III E for the three initial states $|110\rangle,|101\rangle$, and $|011\rangle$. From the data in Fig. 16(a), we see that roughly $1 \%$ of the population in the initial state $|110\rangle$ is transferred to the state $|020\rangle$ after about $10 \mathrm{~ns}$, which is when qubit 1 is tuned through the point where $\omega_{1}+\alpha_{1}=\omega_{0}$, i.e., where the states $|110\rangle$ and $|020\rangle$ are on resonance. Similarly, we observe in Fig. 16(b) that roughly $0.5 \%$ of the population in the initial state $|101\rangle$ is transferred to the state $|200\rangle$ after about $10 \mathrm{~ns}$, which is when qubit 2 is tuned through the point where $\omega_{2}=\omega_{0}+\alpha_{0}$, i.e., where the states $|101\rangle$ and $|200\rangle$ are on resonance. For the case when the initial state is $|011\rangle$, shown in Fig. 16(c), the amount of population lost to states outside the computational subspace during the initial tuning phase is several orders of magnitude less, likely because both qubits 1 and 2, rather than just one of them as in the previous cases, are changing their frequencies rapidly when they are tuned through the resonance $\omega_{1}+\alpha_{1}=\omega_{2}$, where population transfer to the state $|020\rangle$ could occur.

Finally, we note that, for both gates discussed here, the only realistic unwanted spurious transitions are those to states with the same number of excitations. However, there
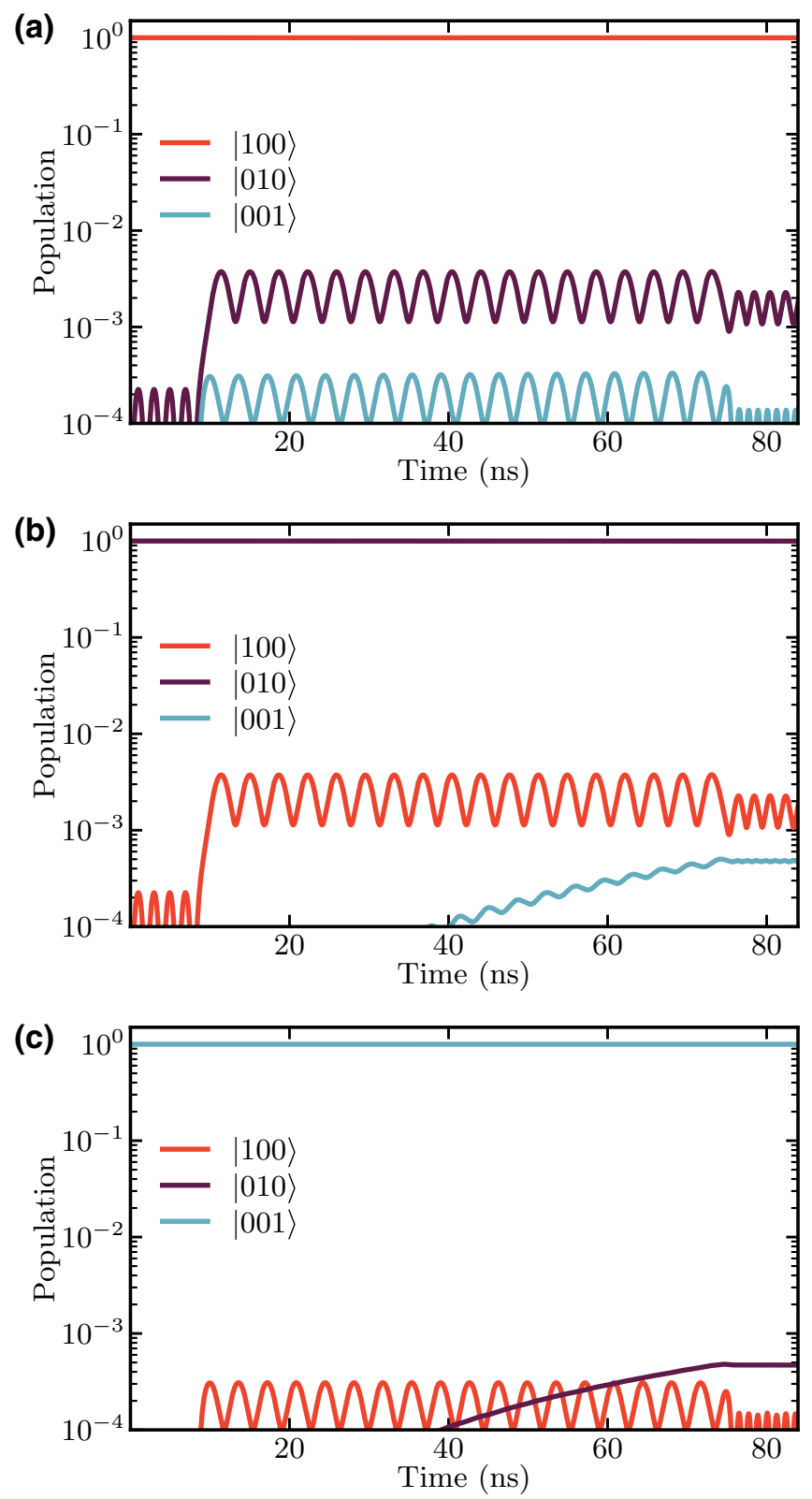

FIG. 15. Population transfers for different initial states [(a) $|100\rangle$, (b) $|010\rangle$, (c) $|001\rangle]$ in the one-excitation subspace in the implementation of the CCZS gate with tunable qubits. All simulation parameters and notation conventions are the same as for Fig. 7 in the main text.

is an additional constraint. The transitions that realistically can come close to being on resonance will not change the number of excitations in any qubit by more than one [see the Hamiltonians in Eqs. (45) and (49)]. Thus, if we begin in the state $|111\rangle$, there is no single process that can take us to, e.g., $|300\rangle$. The only time when one might worry about populating the third excited state of any qubit is thus for the CCZS gate sketched in Fig. 1(b). There, the gate operation includes transitions from $|111\rangle$ to $|201\rangle$ or $|210\rangle$ and back. For these transitions to be active, couplings 

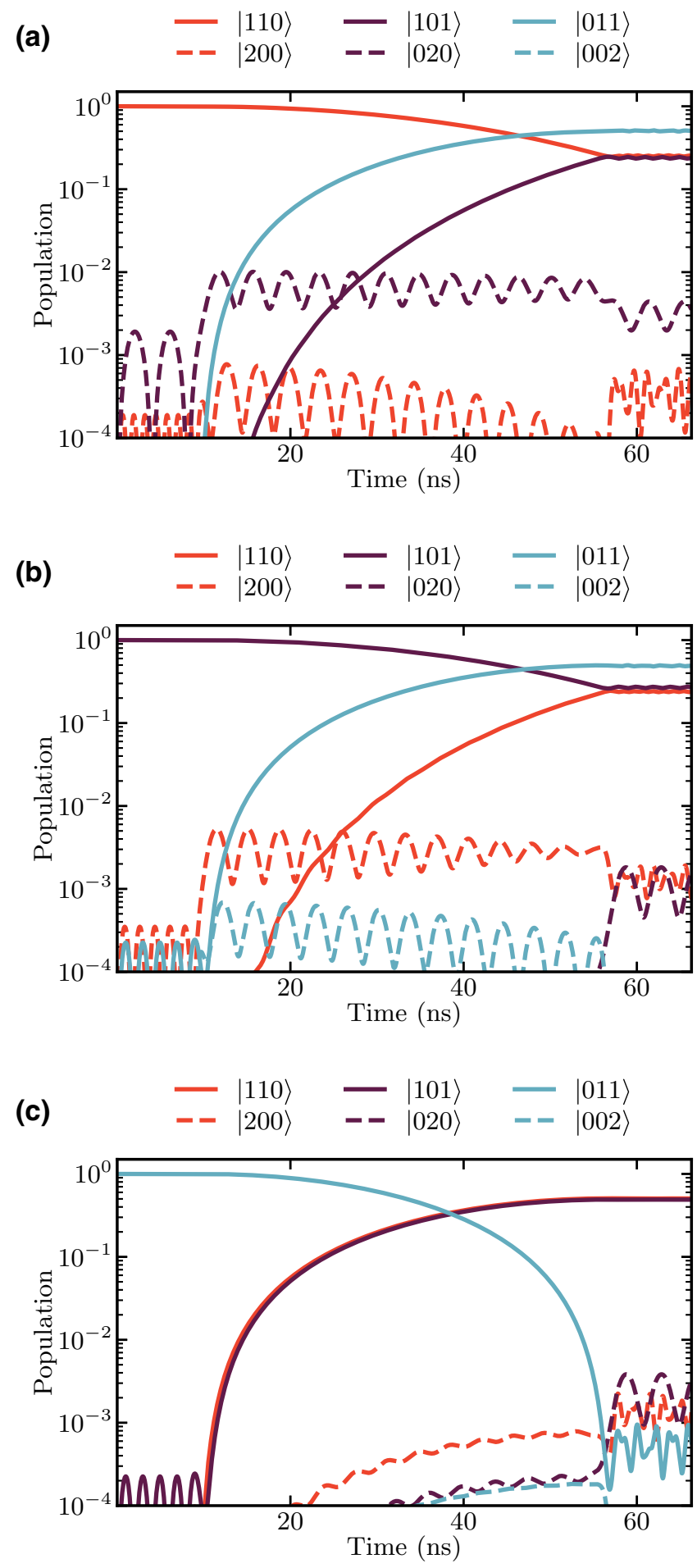

FIG. 16. Population transfers for different initial states [(a) $|110\rangle$, (b) $|101\rangle$, (c) $|011\rangle]$ in the two-excitation subspace in the implementation of the DIV gate with tunable qubits. All simulation parameters and notation conventions are the same as for Fig. 14 in the main text.

are induced between $\left|1_{0} 1_{j}\right\rangle$ and $\left|2_{0} 0_{j}\right\rangle$. The energy difference between these two states is $\left|\omega_{0}+\alpha_{0}-\omega_{j}-\alpha_{j}\right|$. However, the state $|300\rangle$ is separated from the states $|201\rangle$ and $|210\rangle$ by the energy difference $\left|\omega_{0}+2 \alpha_{0}-\omega_{j}-\alpha_{j}\right|$, provided that the qubits can be modeled as anharmonic oscillators with anharmonicities $\alpha_{j}$. These transitions are thus $\alpha_{0}$ away in energy from being activated, which is enough to say that they are far off resonance [they are as far away from the dashed yellow line in Fig. 7(b) as the dashed line therein is from the solid yellow line].

[1] R. P. Feynman, Simulating physics with computers, Int. J. Theor. Phys. 21, 467 (1982).

[2] M. A. Nielsen and I. L. Chuang, Quantum Computation and Quantum Information (Cambridge University Press, Cambridge, 2000).

[3] Y. Cao, J. Romero, J. P. Olson, M. Degroote, P. D. Johnson, M. Kieferová, I. D. Kivlichan, T. Menke, B. Peropadre, N. P. D. Sawaya, S. Sim, L. Veis, and A. AspuruGuzik, Quantum chemistry in the age of Quantum computing, Chem. Rev. 119, 10856 (2019), arXiv:1812.09976.

[4] S. McArdle, S. Endo, A. Aspuru-Guzik, S. C. Benjamin, and X. Yuan, Quantum computational chemistry, Rev. Mod. Phys. 92, 015003 (2020), arXiv:1808.10402.

[5] B. Bauer, S. Bravyi, M. Motta, and G. Kin-Lic Chan, Quantum algorithms for quantum chemistry and Quantum materials science, Chem. Rev. 120, 12685 (2020), arXiv:2001.03685.

[6] R. Orús, S. Mugel, and E. Lizaso, Quantum computing for finance: Overview and prospects, Rev. Phys. 4, 100028 (2019), arXiv:1807.03890.

[7] D. J. Egger, C. Gambella, J. Marecek, S. McFaddin, M. Mevissen, R. Raymond, A. Simonetto, S. Woerner, and E. Yndurain, Quantum computing for finance: State-of-theart and future prospects, IEEE Trans. Quantum Eng. 1, 1 (2020), arXiv:2006.14510.

[8] I. M. Georgescu, S. Ashhab, and F. Nori, Quantum simulation, Rev. Mod. Phys. 86, 153 (2014), arXiv:1308.6253.

[9] A. Montanaro, Quantum algorithms: An overview, Npj Quantum Inf. 2, 15023 (2016), arXiv:1511.04206.

[10] G. Wendin, Quantum information processing with superconducting circuits: A review, Rep. Prog. Phys. 80, 106001 (2017), arXiv:1610.02208.

[11] J. Preskill, Quantum computing in the NISQ era and beyond, Quantum 2, 79 (2018), arXiv:1801.00862.

[12] M. Cerezo, A. Arrasmith, R. Babbush, S. C. Benjamin, S. Endo, K. Fujii, J. R. McClean, K. Mitarai, X. Yuan, L. Cincio, and P. J. Coles, Variational quantum algorithms, Nat. Rev. Phys. 3, 625 (2021), arXiv:2012.09265.

[13] F. Arute et al., Quantum supremacy using a programmable superconducting processor, Nature 574, 505 (2019), arXiv:1911.00577.

[14] J. M. Pino, J. M. Dreiling, C. Figgatt, J. P. Gaebler, S. A. Moses, M. S. Allman, C. H. Baldwin, M. Foss-Feig, D. Hayes, K. Mayer, C. Ryan-Anderson, and B. Neyenhuis, Demonstration of the trapped-ion quantum CCD computer architecture, Nature 592, 209 (2021), arXiv:2003.01293.

[15] G. J. Mooney, G. A. L. White, C. D. Hill, and L. C. L. Hollenberg, Whole-device entanglement in a 65-qubit superconducting quantum computer, arXiv:2102.11521 (2021). 
[16] S. Blinov, B. Wu, and C. Monroe, Comparison of CloudBased Ion Trap and Superconducting Quantum Computer Architectures, arXiv:2102.00371 (2021).

[17] Y. Wu et al., Strong quantum computational advantage using a superconducting quantum processor, arXiv:2106. 14734 (2021).

[18] A. Barenco, C. H. Bennett, R. Cleve, D. P. DiVincenzo, N. Margolus, P. Shor, T. Sleator, J. A. Smolin, and H. Weinfurter, Elementary gates for quantum computation, Phys. Rev. A 52, 3457 (1995), arXiv:9503016 [quant-ph].

[19] J. A. Smolin and D. P. DiVincenzo, Five two-bit quantum gates are sufficient to implement the quantum fredkin gate, Phys. Rev. A 53, 2855 (1996).

[20] L. Isenhower, M. Saffman, and K. Mølmer, Multibit $\mathrm{C}_{k}$ NOT quantum gates via rydberg blockade, Quantum Inf. Process. 10, 755 (2011).

[21] E. Zahedinejad, J. Ghosh, and B. C. Sanders, Designing High-Fidelity Single-Shot Three-Qubit Gates: A Machine-Learning Approach, Phys. Rev. Appl. 6, 054005 (2016), arXiv:1511.08862.

[22] E. Barnes, C. Arenz, A. Pitchford, and S. E. Economou, Fast microwave-driven three-qubit gates for cavitycoupled superconducting qubits, Phys. Rev. B 96, 024504 (2017), arXiv:1612.09384.

[23] P. J. Liebermann, P.-L. Dallaire-Demers, and F. K. Wilhelm, Implementation of the iFREDKIN gate in scalable superconducting architecture for the quantum simulation of Fermionic systems, arXiv:1701.07870 (2017).

[24] T. Liu, B.-Q. Guo, C.-S. Yu, and W.-N. Zhang, Onestep implementation of a hybrid fredkin gate with quantum memories and single superconducting qubit in circuit QED and its applications, Opt. Express 26, 4498 (2018), arXiv:1802.10388.

[25] T. Bækkegaard, L. B. Kristensen, N. J. Loft, C. K. Andersen, D. Petrosyan, and N. T. Zinner, Realization of efficient quantum gates with a superconducting qubit-qutrit circuit, Sci. Rep. 9, 13389 (2019), arXiv:1802.04299.

[26] M. J. Gullans and J. R. Petta, Protocol for a resonantly driven three-qubit toffoli gate with silicon spin qubits, Phys. Rev. B 100, 085419 (2019), arXiv:1905.06756.

[27] S. Daraeizadeh, S. P. Premaratne, N. Khammassi, X. Song, M. Perkowski, and A. Y. Matsuura, Machine-learningbased three-qubit gate design for the toffoli gate and parity check in transmon systems, Phys. Rev. A 102, 012601 (2020), arXiv:1908.01092.

[28] M. Khazali and K. Mølmer, Fast Multiqubit Gates by Adiabatic Evolution in Interacting Excited-State Manifolds of Rydberg Atoms and Superconducting Circuits, Phys. Rev. X 10, 021054 (2020), arXiv:2006.07035.

[29] N. J. S. Loft, M. Kjaergaard, L. B. Kristensen, C. K. Andersen, T. W. Larsen, S. Gustavsson, W. D. Oliver, and N. T. Zinner, Quantum interference device for controlled two-qubit operations, Npj Quantum Inf. 6, 47 (2020), arXiv: 1809.09049 .

[30] S. E. Rasmussen, K. Groenland, R. Gerritsma, K. Schoutens, and N. T. Zinner, Single-step implementation of high-fidelity $n$-bit toffoli gates, Phys. Rev. A 101, 022308 (2020), arXiv:1910.07548.

[31] S. E. Rasmussen and N. T. Zinner, Simple implementation of high fidelity controlled-iSWAP gates and quantum circuit exponentiation of non-hermitian gates, Phys. Rev. Res. 2, 033097 (2020), arXiv:2002.11728.

[32] J. D. A. Espinoza, K. Groenland, M. Mazzanti, K. Schoutens, and R. Gerritsma, A high-fidelity method for a single-step $N$-bit Toffoli gate in trapped ions, arXiv:2010.08490 (2020).

[33] J. T. Young, P. Bienias, R. Belyansky, A. M. Kaufman, and A. V. Gorshkov, Asymmetric blockade and multi-qubit gates via dipole-dipole interactions, arXiv:2006.02486 (2020).

[34] D. Yu, W. Zhang, J.-m. Liu, S. Su, and J. Qian, Spheroidalstructure-based multi-qubit Toffoli gate via asymmetric Rydberg interaction, arXiv:2007.11938 (2020).

[35] P. Zhao, P. Xu, D. Lan, X. Tan, H. Yu, and Y. Yu, Switchable Next-Nearest-Neighbor Coupling for Controlled Two-Qubit Operations, Phys. Rev. Appl. 14, 064016 (2020), arXiv:2004.08639.

[36] Y. He, J.-X. Liu, F. Q. Guo, L.-L. Yan, R. Luo, E. Liang, S.-L. Su, and M. Feng, Multiple-qubit Rydberg quantum logic gate via dressed-states scheme, arXiv:2010.14704 (2020).

[37] E. Bahnsen, S. E. Rasmussen, N. J. S. Loft, and N. T. Zinner, Application of the Diamond Gate in Quantum Fourier Transformations and Quantum Machine Learning, arXiv:2103.08605 (2021).

[38] T. Monz, K. Kim, W. Hänsel, M. Riebe, A. S. Villar, P. Schindler, M. Chwalla, M. Hennrich, and R. Blatt, Realization of the Quantum Toffoli Gate with Trapped Ions, Phys. Rev. Lett. 102, 040501 (2009), arXiv:0804.0082.

[39] M. D. Reed, L. DiCarlo, S. E. Nigg, L. Sun, L. Frunzio, S. M. Girvin, and R. J. Schoelkopf, Realization of three-qubit quantum error correction with superconducting circuits, Nature 482, 382 (2012).

[40] R. B. Patel, J. Ho, F. Ferreyrol, T. C. Ralph, and G. J. Pryde, A quantum fredkin gate, Sci. Adv. 2, e1501531 (2016).

[41] H. Levine, A. Keesling, G. Semeghini, A. Omran, T. T. Wang, S. Ebadi, H. Bernien, M. Greiner, V. Vuletić, H. Pichler, and M. D. Lukin, Parallel Implementation of High-Fidelity Multiqubit Gates with Neutral Atoms, Phys. Rev. Lett. 123, 170503 (2019), arXiv:1908.06101.

[42] T. Roy, S. Hazra, S. Kundu, M. Chand, M. P. Patankar, and R. Vijay, Programmable Superconducting Processor with Native Three-Qubit Gates, Phys. Rev. Appl. 14, 014072 (2020), arXiv:1809.00668.

[43] S. Ru, Y. Wang, M. An, F. Wang, P. Zhang, and F. $\mathrm{Li}$, Realization of a deterministic quantum toffoli gate with a single photon, Phys. Rev. A 103, 022606 (2021), arXiv:2010.14776.

[44] A. Mezzacapo, L. Lamata, S. Filipp, and E. Solano, Many-Body Interactions with Tunable-Coupling Transmon Qubits, Phys. Rev. Lett. 113, 050501 (2014), arXiv:1403.3652.

[45] D. Kafri, C. Quintana, Y. Chen, A. Shabani, J. M. Martinis, and H. Neven, Tunable inductive coupling of superconducting qubits in the strongly nonlinear regime, Phys. Rev. A 95, 052333 (2017), arXiv:1606.08382.

[46] N. Chancellor, S. Zohren, and P. A. Warburton, Circuit design for multi-body interactions in superconducting quantum annealing systems with applications to a 
scalable architecture, Npj Quantum Inf. 3, 21 (2017), arXiv:1603.09521.

[47] M. Kounalakis, Y. M. Blanter, and G. A. Steele, Synthesizing multi-phonon quantum superposition states using flux-mediated three-body interactions with superconducting qubits, npj Quantum Inf. 5, 100 (2019), arXiv:1905.10225.

[48] S. P. Pedersen, K. S. Christensen, and N. T. Zinner, Native three-body interaction in superconducting circuits, Phys. Rev. Res. 1, 033123 (2019), arXiv:1910.08741.

[49] M. Schöndorf and F. Wilhelm, Nonpairwise Interactions Induced by Virtual Transitions in Four Coupled Artificial Atoms, Phys. Rev. Appl. 12, 064026 (2019), arXiv: 1811.07683.

[50] F. Petiziol, M. Sameti, S. Carretta, S. Wimberger, and F. Mintert, Quantum simulation of three-body interactions in weakly driven quantum systems, arXiv:2011.03399 (2020).

[51] M. A. Rol, L. Ciorciaro, F. K. Malinowski, B. M. Tarasinski, R. E. Sagastizabal, C. C. Bultink, Y. Salathe, N. Haandbaek, J. Sedivy, and L. DiCarlo, Time-domain characterization and correction of on-chip distortion of control pulses in a quantum processor, Appl. Phys. Lett. 116, 054001 (2020), arXiv:1907.04818.

[52] X. Gu, A. F. Kockum, A. Miranowicz, Y.-X. Liu, and F. Nori, Microwave photonics with superconducting quantum circuits, Phys. Rep. 718-719, 1 (2017), arXiv:1707.02046.

[53] P. Krantz, M. Kjaergaard, F. Yan, T. P. Orlando, S. Gustavsson, and W. D. Oliver, A quantum engineer's guide to superconducting qubits, Appl. Phys. Rev. 6, 021318 (2019), arXiv:1904.06560.

[54] A. F. Kockum and F. Nori, in Fundamentals and Frontiers of the Josephson Effect, edited by F. Tafuri (Springer, 2019), p. 703 arXiv:1908.09558.

[55] M. Kjaergaard, M. E. Schwartz, J. Braumüller, P. Krantz, J. I.-J. Wang, S. Gustavsson, and W. D. Oliver, Superconducting qubits: Current state of play, Annu. Rev. Condens. Matter Phys. 11, 369 (2020), arXiv:1905. 13641.

[56] A. Blais, A. L. Grimsmo, S. M. Girvin, and A. Wallraff, Circuit Quantum Electrodynamics, arXiv:2005.12667 (2020).

[57] A. Bermudez, X. Xu, R. Nigmatullin, J. O'Gorman, V. Negnevitsky, P. Schindler, T. Monz, U. G. Poschinger, C. Hempel, J. Home, F. Schmidt-Kaler, M. Biercuk, R. Blatt, S. Benjamin, and M. Müller, Assessing the Progress of Trapped-Ion Processors Towards Fault-Tolerant Quantum Computation, Phys. Rev. X 7, 041061 (2017), arXiv: 1705.02771.

[58] J. D. Wong-Campos, S. A. Moses, K. G. Johnson, and C. Monroe, Demonstration of Two-Atom Entanglement with Ultrafast Optical Pulses, Phys. Rev. Lett. 119, 230501 (2017), arXiv:1709.05179.

[59] V. M. Schäfer, C. J. Ballance, K. Thirumalai, L. J. Stephenson, T. G. Ballance, A. M. Steane, and D. M. Lucas, Fast quantum logic gates with trapped-ion qubits, Nature 555, 75 (2018), arXiv:1709.06952.

[60] C. D. Bruzewicz, J. Chiaverini, R. McConnell, and J. M. Sage, Trapped-ion quantum computing: Progress and challenges, Appl. Phys. Rev. 6, 021314 (2019), arXiv:1904.04178.

[61] C. Zhang, F. Pokorny, W. Li, G. Higgins, A. Pöschl, I. Lesanovsky, and M. Hennrich, Submicrosecond entangling gate between trapped ions via rydberg interaction, Nature 580, 345 (2020), arXiv:1908.11284.

[62] I. Pogorelov, T. Feldker, C. D. Marciniak, L. Postler, G. Jacob, O. Kriegelsteiner, V. Podlesnic, M. Meth, V. Negnevitsky, M. Stadler, B. Höfer, C. Wächter, K. Lakhmanskiy, R. Blatt, P. Schindler, and T. Monz, A compact iontrap quantum computing demonstrator, arXiv:2101.11390 (2021).

[63] D. Jaksch, J. I. Cirac, P. Zoller, S. L. Rolston, R. Côté, and M. D. Lukin, Fast Quantum Gates for Neutral Atoms, Phys. Rev. Lett. 85, 2208 (2000), arXiv:0004038 [quan$\mathrm{t}-\mathrm{ph}$.

[64] M. Saffman, T. G. Walker, and K. Mølmer, Quantum information with rydberg atoms, Rev. Mod. Phys. 82, 2313 (2010), arXiv:0909.4777.

[65] K. M. Maller, M. T. Lichtman, T. Xia, Y. Sun, M. J. Piotrowicz, A. W. Carr, L. Isenhower, and M. Saffman, Rydberg-blockade controlled-not gate and entanglement in a two-dimensional array of neutral-atom qubits, Phys. Rev. A 92, 022336 (2015).

[66] M. Saffman, Quantum computing with atomic qubits and rydberg interactions: Progress and challenges, J. Phys. B: At. Mol. Opt. Phys. 49, 202001 (2016), arXiv:1605.05207.

[67] A. Browaeys, D. Barredo, and T. Lahaye, Experimental investigations of dipole-dipole interactions between a few rydberg atoms, J. Phys. B: At. Mol. Opt. Phys. 49, 152001 (2016).

[68] F. W. Strauch, P. R. Johnson, A. J. Dragt, C. J. Lobb, J. R. Anderson, and F. C. Wellstood, Quantum Logic Gates for Coupled Superconducting Phase Qubits, Phys. Rev. Lett. 91, 167005 (2003).

[69] N. Schuch and J. Siewert, Natural two-qubit gate for quantum computation using the $\$ \mathrm{XY} \$$ interaction, Phys. Rev. A 67, 032301 (2003).

[70] P.-L. Dallaire-Demers and F. K. Wilhelm, Quantum gates and architecture for the quantum simulation of the fermi-hubbard model, Phys. Rev. A 94, 062304 (2016), arXiv: 1606.00208 .

[71] D. M. Greenberger, M. A. Horne, and A. Zeilinger, in Bell's Theorem, Quantum Theory and Conceptions of the Universe, edited by M. Kafatos (Springer Netherlands, Dordrecht, 1989), p. 69, arXiv:0712.0921.

[72] D. Bouwmeester, J.-W. Pan, M. Daniell, H. Weinfurter, and A. Zeilinger, Observation of Three-Photon Greenberger-Horne-Zeilinger Entanglement, Phys. Rev. Lett. 82, 1345 (1999).

[73] R. H. Dicke, Coherence in spontaneous radiation processes, Phys. Rev. 93, 99 (1954).

[74] S. Haroche and J.-M. Raimond, Exploring the Quantum (Oxford University Press, Oxford, 2006).

[75] N. Shammah, S. Ahmed, N. Lambert, S. De Liberato, and F. Nori, Open quantum systems with local and collective incoherent processes: Efficient numerical simulations using permutational invariance, Phys. Rev. A 98, 063815 (2018), arXiv:1805.05129. 
[76] A. Y. Kitaev, A. Shen, and M. N. Vyalyi, Classical and Quantum Computation (American Mathematical Society, Providence, Rhode Island, 2002).

[77] D. Poulin, A. Kitaev, D. S. Steiger, M. B. Hastings, and M. Troyer, Quantum Algorithm for Spectral Measurement with a Lower Gate Count, Phys. Rev. Lett. 121, 010501 (2018), arXiv:1711.11025.

[78] R. Babbush, C. Gidney, D. W. Berry, N. Wiebe, J. McClean, A. Paler, A. Fowler, and H. Neven, Encoding Electronic Spectra in Quantum Circuits with Linear T Complexity, Phys. Rev. X 8, 041015 (2018), arXiv:1805.03662.

[79] G. H. Low and I. L. Chuang, Hamiltonian simulation by qubitization, Quantum 3, 163 (2019), arXiv:1610.06546.

[80] A. Gilyén, Y. Su, G. H. Low, and N. Wiebe, in Proceedings of the 51st Annual ACM SIGACT Symposium on Theory of Computing (ACM, New York, NY, USA, 2019), p. 193, arXiv:1806.01838.

[81] J. M. Martyn, Z. M. Rossi, A. K. Tan, and I. L. Chuang, A Grand Unification of Quantum Algorithms, arXiv:2105.02859 (2021).

[82] X. Gu, J. Allcock, S. An, and Y.-X. Liu, Efficient multiqubit subspace rotations via topological quantum walks, arXiv:2111.06534 (2021).

[83] H. Buhrman, R. Cleve, J. Watrous, and R. de Wolf, Quantum Fingerprinting, Phys. Rev. Lett. 87, 167902 (2001), arXiv:0102001 [quant-ph].

[84] J. Zhao, Y.-H. Zhang, C.-P. Shao, Y.-C. Wu, G.-C. Guo, and G.-P. Guo, Building quantum neural networks based on a swap test, Phys. Rev. A 100, 012334 (2019), arXiv:1904.12697.

[85] P. K. Barkoutsos, J. F. Gonthier, I. Sokolov, N. Moll, G. Salis, A. Fuhrer, M. Ganzhorn, D. J. Egger, M. Troyer, A. Mezzacapo, S. Filipp, and I. Tavernelli, Quantum algorithms for electronic structure calculations: Particle-hole Hamiltonian and optimized wave-function expansions, Phys. Rev. A 98, 022322 (2018), arXiv:1805.04340.

[86] S. Hadfield, Z. Wang, B. O'Gorman, E. Rieffel, D. Venturelli, and R. Biswas, From the quantum approximate optimization algorithm to a quantum alternating operator ansatz, Algorithms 12, 34 (2019), arXiv:1709.03489.

[87] M. Streif, M. Leib, F. Wudarski, E. Rieffel, and Z. Wang, Quantum algorithms with local particle-number conservation: Noise effects and error correction, Phys. Rev. A 103, 042412 (2021), arXiv:2011.06873.

[88] R. C. Bialczak, M. Ansmann, M. Hofheinz, E. Lucero, M. Neeley, A. D. O'Connell, D. Sank, H. Wang, J. Wenner, M. Steffen, A. N. Cleland, and J. M. Martinis, Quantum process tomography of a universal entangling gate implemented with josephson phase qubits, Nat. Phys. 6, 409 (2010).

[89] R. Barends et al., Diabatic Gates for Frequency-Tunable Superconducting Qubits, Phys. Rev. Lett. 123, 210501 (2019), arXiv:1907.02510.

[90] C. K. Andersen, A. Remm, S. Lazar, S. Krinner, J. Heinsoo, J.-C. Besse, M. Gabureac, A. Wallraff, and C. Eichler, Entanglement stabilization using ancilla-based parity detection and real-time feedback in superconducting circuits, Npj Quantum Inf. 5, 69 (2019), arXiv:1902.06946.

[91] V. Negîrneac, H. Ali, N. Muthusubramanian, F. Battistel, R. Sagastizabal, M. S. Moreira, J. F. Marques,
W. J. Vlothuizen, M. Beekman, C. Zachariadis, N. Haider, A. Bruno, and L. DiCarlo, High-Fidelity Controlled-Z Gate with Maximal Intermediate Leakage Operating at the Speed Limit in a Superconducting Quantum Processor, Phys. Rev. Lett. 126, 220502 (2021), arXiv:2008.07411.

[92] Y. Sung, L. Ding, J. Braumüller, A. Vepsäläinen, B. Kannan, M. Kjaergaard, A. Greene, G. O. Samach, C. McNally, D. Kim, A. Melville, B. M. Niedzielski, M. E. Schwartz, J. L. Yoder, T. P. Orlando, S. Gustavsson, and W. D. Oliver, Realization of High-Fidelity CZ and ZZFree iSWAP Gates with a Tunable Coupler, Phys. Rev. X 11, 021058 (2021), arXiv:2011.01261.

[93] B. Foxen et al., Demonstrating a Continuous Set of TwoQubit Gates for Near-Term Quantum Algorithms, Phys. Rev. Lett. 125, 120504 (2020), arXiv:2001.08343.

[94] H. Xu, W. Liu, Z. Li, J. Han, J. Zhang, K. Linghu, Y. Li, M. Chen, Z. Yang, J. Wang, T. Ma, G. Xue, Y. Jin, and H. Yu, Realization of adiabatic and diabatic $\mathrm{CZ}$ gates in superconducting qubits coupled with a tunable coupler, Chin. Phys. B 30, 044212 (2021), arXiv:2010.14053.

[95] D. C. McKay, S. Filipp, A. Mezzacapo, E. Magesan, J. M. Chow, and J. M. Gambetta, Universal Gate for FixedFrequency Qubits via a Tunable Bus, Phys. Rev. Appl. 6, 064007 (2016), arXiv:1604.03076.

[96] M. Roth, M. Ganzhorn, N. Moll, S. Filipp, G. Salis, and S. Schmidt, Analysis of a parametrically driven exchangetype gate and a two-photon excitation gate between superconducting qubits, Phys. Rev. A 96, 062323 (2017), arXiv:1708.02090.

[97] A. Bengtsson, P. Vikstål, C. Warren, M. Svensson, X. Gu, A. F. Kockum, P. Krantz, C. Križan, D. Shiri, I.-M. Svensson, G. Tancredi, G. Johansson, P. Delsing, G. Ferrini, and J. Bylander, Improved Success Probability with Greater Circuit Depth for the Quantum Approximate Optimization Algorithm, Phys. Rev. Appl. 14, 034010 (2020), arXiv:1912.10495.

[98] M. Ganzhorn, G. Salis, D. J. Egger, A. Fuhrer, M. Mergenthaler, C. Müller, P. Müller, S. Paredes, M. Pechal, M. Werninghaus, and S. Filipp, Benchmarking the noise sensitivity of different parametric two-qubit gates in a single superconducting quantum computing platform, Phys. Rev. Res. 2, 033447 (2020), arXiv:2005.05696.

[99] E. Sjöqvist, Nonadiabatic holonomic single-qubit gates in off-resonant $\Lambda$ systems, Phys. Lett. A 380, 65 (2016), arXiv:1511.00911.

[100] E. Sjöqvist, D. M. Tong, L. M. Andersson, B. Hessmo, M. Johansson, and K. Singh, Non-adiabatic holonomic quantum computation, New J. Phys. 14, 103035 (2012), arXiv: 1107.5127.

[101] E. Fredkin and T. Toffoli, Conservative logic, Int. J. Theor. Phys. 21, 219 (1982).

[102] G. J. Milburn, Quantum Optical Fredkin Gate, Phys. Rev. Lett. 62, 2124 (1989).

[103] J. Koch, T. M. Yu, J. Gambetta, A. A. Houck, D. I. Schuster, J. Majer, A. Blais, M. H. Devoret, S. M. Girvin, and R. J. Schoelkopf, Charge-insensitive qubit design derived from the cooper pair box, Phys. Rev. A 76, 042319 (2007), arXiv:0703002 [cond-mat].

[104] A. A. Abdumalikov Jr, J. M. Fink, K. Juliusson, M. Pechal, S. Berger, A. Wallraff, S. Filipp, A. A. Abdumalikov, J. M. Fink, K. Juliusson, M. Pechal, S. Berger, A. 
Wallraff, and S. Filipp, Experimental realization of nonabelian non-adiabatic geometric gates, Nature 496, 482 (2013), arXiv:1304.5186.

[105] S. Danilin, A. Vepsäläinen, and G. S. Paraoanu, Experimental state control by fast non-abelian holonomic gates with a superconducting qutrit, Phys. Scr. 93, 055101 (2018), arXiv:1804.01759.

[106] D. J. Egger, M. Ganzhorn, G. Salis, A. Fuhrer, P. Müller, P. K. Barkoutsos, N. Moll, I. Tavernelli, and S. Filipp, Entanglement Generation in Superconducting Qubits Using Holonomic Operations, Phys. Rev. Appl. 11, 014017 (2019), arXiv:1804.04900.

[107] M. Ganzhorn, D. J. Egger, P. Barkoutsos, P. Ollitrault, G. Salis, N. Moll, M. Roth, A. Fuhrer, P. Mueller, S. Woerner, I. Tavernelli, and S. Filipp, Gate-Efficient Simulation of Molecular Eigenstates on a Quantum Computer, Phys. Rev. Appl. 11, 044092 (2019), arXiv:1809.05057.

[108] D. M. Abrams, N. Didier, B. R. Johnson, M. P. da Silva, and C. A. Ryan, Implementation of XY entangling gates with a single calibrated pulse, Nat. Electron. 3, 744 (2020), arXiv: 1912.04424.

[109] T. Toffoli, in Automata, Languages and Programming, Lecture Notes in Computer Science, Vol. 85, edited by J. de Bakker and J. van Leeuwen (Springer Berlin Heidelberg, Berlin, Heidelberg, 1980).

[110] A. Fedorov, L. Steffen, M. Baur, M. P. da Silva, and A. Wallraff, Implementation of a Toffoli gate with superconducting circuits, Nature 481, 170 (2012), arXiv:1108.3966.

[111] R. Horodecki, P. Horodecki, M. Horodecki, and K. Horodecki, Quantum entanglement, Rev. Mod. Phys. 81, 865 (2009), arXiv:0702225 [quant-ph].

[112] H. J. Briegel, D. E. Browne, W. Dür, R. Raussendorf, and M. Van den Nest, Measurement-based quantum computation, Nat. Phys. 5, 19 (2009), arXiv:0910.1116.

[113] J. Preskill, Quantum computing and the entanglement frontier, arXiv:1203.5813 (2012).

[114] N. Gisin and R. Thew, Quantum communication, Nat. Photonics 1, 165 (2007), arXiv:0703255 [quant-ph].

[115] Y. Wang, Y. Li, Z.-Q. Yin, and B. Zeng, 16-qubit IBM universal quantum computer can be fully entangled, Npj Quantum Inf. 4, 46 (2018), arXiv:1801.03782.

[116] G. J. Mooney, C. D. Hill, and L. C. L. Hollenberg, Entanglement in a 20-qubit superconducting quantum computer, Sci. Rep. 9, 13465 (2019), arXiv:1903.11747.

[117] K. X. Wei, I. Lauer, S. Srinivasan, N. Sundaresan, D. T. McClure, D. Toyli, D. C. McKay, J. M. Gambetta, and S. Sheldon, Verifying multipartite entangled greenbergerhorne-Zeilinger states via multiple quantum coherences, Phys. Rev. A 101, 032343 (2020), arXiv:1905.05720.

[118] B. Yang, R. Raymond, H. Imai, H. Chang, and H. Hiraishi, Testing Scalable Bell Inequalities for Quantum Graph States on IBM Quantum Devices, arXiv:2101.10307 (2021).

[119] N. Friis, O. Marty, C. Maier, C. Hempel, M. Holzäpfel, P. Jurcevic, M. B. Plenio, M. Huber, C. Roos, R. Blatt, and B. Lanyon, Observation of Entangled States of a Fully Controlled 20-Qubit System, Phys. Rev. X 8, 021012 (2018), arXiv:1711.11092.
[120] A. Omran, H. Levine, A. Keesling, G. Semeghini, T. T. Wang, S. Ebadi, H. Bernien, A. S. Zibrov, H. Pichler, S. Choi, J. Cui, M. Rossignolo, P. Rembold, S. Montangero, T. Calarco, M. Endres, M. Greiner, V. Vuletić, and M. D. Lukin, Generation and manipulation of Schrödinger cat states in rydberg atom arrays, Science 365, 570 (2019), arXiv:1905.05721.

[121] M. Neeley, R. C. Bialczak, M. Lenander, E. Lucero, M. Mariantoni, A. D. O'Connell, D. Sank, H. Wang, M. Weides, J. Wenner, Y. Yin, T. Yamamoto, A. N. Cleland, and J. M. Martinis, Generation of three-qubit entangled states using superconducting phase qubits, Nature 467, 570 (2010), arXiv:1004.4246.

[122] W. Dür, G. Vidal, and J. I. Cirac, Three qubits can be entangled in two inequivalent ways, Phys. Rev. A 62, 062314 (2000), arXiv:0005115 [quant-ph].

[123] L. Pezzè, A. Smerzi, M. K. Oberthaler, R. Schmied, and P. Treutlein, Quantum metrology with nonclassical states of atomic ensembles, Rev. Mod. Phys. 90, 035005 (2018), arXiv:1609.01609.

[124] V. Paulisch, M. Perarnau-Llobet, A. González-Tudela, and J. I. Cirac, Quantum metrology with one-dimensional superradiant photonic states, Phys. Rev. A 99, 043807 (2019), arXiv:1805.00712.

[125] R. Prevedel, G. Cronenberg, M. S. Tame, M. Paternostro, P. Walther, M. S. Kim, and A. Zeilinger, Experimental Realization of Dicke States of up to Six Qubits for Multiparty Quantum Networking, Phys. Rev. Lett. 103, 020503 (2009), arXiv:0903.2212.

[126] W. Wieczorek, R. Krischek, N. Kiesel, P. Michelberger, G. Tóth, and H. Weinfurter, Experimental Entanglement of a Six-Photon Symmetric Dicke State, Phys. Rev. Lett. 103, 020504 (2009).

[127] A. Chiuri, C. Greganti, M. Paternostro, G. Vallone, and P. Mataloni, Experimental Quantum Networking Protocols via Four-Qubit Hyperentangled Dicke States, Phys. Rev. Lett. 109, 173604 (2012).

[128] J. Miguel-Ramiro and W. Dür, Delocalized information in quantum networks, New J. Phys. 22, 043011 (2020), arXiv:1912.12935.

[129] J. Cook, S. Eidenbenz, and A. Bartschi, in 2020 IEEE International Conference on Quantum Computing and Engineering (QCE) (IEEE, 2020), p. 83, arXiv:1910.13483.

[130] A. Bartschi and S. Eidenbenz, in 2020 IEEE International Conference on Quantum Computing and Engineering (QCE) (IEEE, 2020), p. 72, arXiv:2006. 00354.

[131] D. B. Hume, C. W. Chou, T. Rosenband, and D. J. Wineland, Preparation of dicke states in an ion chain, Phys. Rev. A 80, 052302 (2009), arXiv:0909.0046.

[132] J. K. Stockton, R. van Handel, and H. Mabuchi, Deterministic dicke-state preparation with continuous measurement and control, Phys. Rev. A 70, 022106 (2004), arXiv:0402137 [quant-ph].

[133] Y.-F. Xiao, X.-B. Zou, and G.-C. Guo, Generation of atomic entangled states with selective resonant interaction in cavity quantum electrodynamics, Phys. Rev. A 75, 012310 (2007). 
[134] L. Lamata, C. E. López, B. P. Lanyon, T. Bastin, J. C. Retamal, and E. Solano, Deterministic generation of arbitrary symmetric states and entanglement classes, Phys. Rev. A 87, 032325 (2013), arXiv:1211.0404.

[135] S. S. Ivanov, N. V. Vitanov, and N. V. Korolkova, Creation of arbitrary dicke and NOON states of trapped-ion qubits by global addressing with composite pulses, New J. Phys. 15, 023039 (2013).

[136] C. Wu, C. Guo, Y. Wang, G. Wang, X.-L. Feng, and J.-L. Chen, Generation of dicke states in the ultrastrongcoupling regime of circuit QED systems, Phys. Rev. A 95, 013845 (2017).

[137] K. Chakraborty, B.-S. Choi, A. Maitra, and S. Maitra, Efficient quantum algorithms to construct arbitrary dicke states, Quantum Inf. Process. 13, 2049 (2014), arXiv:1209.5932.

[138] A. Bärtschi and S. Eidenbenz, in Lecture Notes in Computer Science (including subseries Lecture Notes in Artificial Intelligence and Lecture Notes in Bioinformatics), Vol. 11651 LNCS (2019), p. 126, arXiv:1904.07358.

[139] C. S. Mukherjee, S. Maitra, V. Gaurav, and D. Roy, On Actual Preparation of Dicke State on a Quantum Computer, arXiv:2007.01681 (2020).

[140] M. Tavis and F. W. Cummings, Exact solution for an N-molecule-radiation-Field Hamiltonian, Phys. Rev. 170, 379 (1968).

[141] A. B. Klimov and S. M. Chumakov, A Group-Theoretical Approach to Quantum Optics (Wiley, Weinheim, 2009).

[142] A. Noguchi, K. Toyoda, and S. Urabe, Generation of Dicke States with Phonon-Mediated Multilevel Stimulated Raman Adiabatic Passage, Phys. Rev. Lett. 109, 260502 (2012).

[143] R. G. Unanyan, B. W. Shore, and K. Bergmann, Laserdriven population transfer in four-level atoms: Consequences of non-abelian geometrical adiabatic phase factors, Phys. Rev. A 59, 2910 (1999).

[144] V. A. Mousolou and E. Sjöqvist, Entangling power of holonomic gates in atom-based systems, J. Phys. A: Math. Theor. 51, 475303 (2018), arXiv:1803.00308.

[145] T. Kobayashi, R. Ikuta, S. K. Özdemir, M. Tame, T. Yamamoto, M. Koashi, and N. Imoto, Universal gates for transforming multipartite entangled dicke states, New J. Phys. 16, 023005 (2014).

[146] L. DiCarlo, J. M. Chow, J. M. Gambetta, L. S. Bishop, B. R. Johnson, D. I. Schuster, J. Majer, A. Blais, L. Frunzio, S. M. Girvin, and R. J. Schoelkopf, Demonstration of two-qubit algorithms with a superconducting quantum processor, Nature 460, 240 (2009), arXiv:0903.2030.

[147] M. Reagor et al., Demonstration of universal parametric entangling gates on a multi-qubit lattice, Sci. Adv. 4, eaao3603 (2018), arXiv: 1706.06570.

[148] S. A. Caldwell et al., Parametrically Activated Entangling Gates Using Transmon Qubits, Phys. Rev. Appl. 10, 034050 (2018), arXiv:1706.06562.

[149] M. A. Nielsen, A simple formula for the average gate fidelity of a quantum dynamical operation, Phys. Lett. A 303, 249 (2002), arXiv:0205035 [quant-ph].
[150] D. Willsch, Ph.D. thesis, RWTH Aachen University, 2020, arXiv:2008.13490.

[151] J. R. Johansson, P. D. Nation, and F. Nori, QuTiP: An open-source python framework for the dynamics of open quantum systems, Comput. Phys. Commun. 183, 1760 (2012), arXiv:1110.0573.

[152] J. R. Johansson, P. D. Nation, and F. Nori, QuTiP 2: A python framework for the dynamics of open quantum systems, Comput. Phys. Commun. 184, 1234 (2013), arXiv: 1211.6518

[153] B.-J. Liu, X.-K. Song, Z.-Y. Xue, X. Wang, and M.-H. Yung, Plug-And-Play Approach to Nonadiabatic Geometric Quantum Gates, Phys. Rev. Lett. 123, 100501 (2019), arXiv:1806.07904.

[154] H. Abraham et al., Qiskit: An open-source framework for quantum computing, (2019).

[155] M. G. Neeley, Ph.D. thesis, University of California Santa Barbara, 2010.

[156] A. Galiautdinov and J. M. Martinis, Maximally entangling tripartite protocols for josephson phase qubits, Phys. Rev. A 78, 010305 (2008), arXiv:0804.3159.

[157] C. Rigetti and M. Devoret, Fully microwave-tunable universal gates in superconducting qubits with linear couplings and fixed transition frequencies, Phys. Rev. B 81, 134507 (2010).

[158] J. M. Chow, A. D. Córcoles, J. M. Gambetta, C. Rigetti, B. R. Johnson, J. A. Smolin, J. R. Rozen, G. A. Keefe, M. B. Rothwell, M. B. Ketchen, and M. Steffen, Simple All-Microwave Entangling Gate for Fixed-Frequency Superconducting Qubits, Phys. Rev. Lett. 107, 080502 (2011), arXiv:1106.0553.

[159] E. Farhi, J. Goldstone, and S. Gutmann, A Quantum Approximate Optimization Algorithm, arXiv:1411.4028 (2014).

[160] A. Peruzzo, J. McClean, P. Shadbolt, M.-H. Yung, X.-Q. Zhou, P. J. Love, A. Aspuru-Guzik, and J. L. O'Brien, A variational eigenvalue solver on a photonic quantum processor, Nat. Commun. 5, 4213 (2014).

[161] A. Kay, Tutorial on the Quantikz package, arXiv:1809. 03842 (2018).

[162] Y. Niu, V. Smelyanskiy, and S. Boixo Castrillo, Three Qubit Entangling Gate Through Two-Local Hamiltonian Control, (2020), US Patent Application PCT/US2019/016047.

[163] Y. Kim, A. Morvan, L. B. Nguyen, R. K. Naik, C. Jünger, L. Chen, J. M. Kreikebaum, D. I. Santiago, and I. Siddiqi, High-fidelity iToffoli gate for fixedfrequency superconducting qubits, arXiv:2108.10288 (2021).

[164] M. Roth, N. Moll, G. Salis, M. Ganzhorn, D. J. Egger, S. Filipp, and S. Schmidt, Adiabatic quantum simulations with driven superconducting qubits, Phys. Rev. A 99, 022323 (2019), arXiv:1808.04666.

[165] S. J. Buckle, S. M. Barnett, P. L. Knight, M. A. Lauder, and D. T. Pegg, Atomic interferometers, Optica Acta: Int. J. Opt. 33, 1129 (1986). 